\title{
Existence of stable standing waves and instability of standing waves to a class of quasilinear Schrödinger equations with potential
}

\author{
Jianqing Chen and Eugénio M. Rocha
}

Communicated by Y. Charles Li, received Feburary 27, 2011.

\begin{abstract}
For a class of quasilinear Schrödinger equations with harmonic potential of the form

$$
i \varphi_{t}=-\triangle \varphi+|x|^{2} \varphi-|\varphi|^{p-1} \varphi-2\left(\triangle|\varphi|^{2}\right) \varphi, \quad t \geq 0, x \in \mathbb{R}^{N},
$$

we prove firstly the existence of stable standing waves for $1<p<3+\frac{4}{N}$ and then study the instability of standing waves for $3+\frac{4}{N} \leq p<\frac{3 N+2}{N-2}$. Our results indicate that the quasilinear term $\left(\triangle|\varphi|^{2}\right) \varphi$ makes the standing waves more stable than their counterpart in the semilinear case, which is consistent with the physical phenomena and is in striking contrast with the classical semilinear Schrödinger equations with potential.
\end{abstract}

\section{CONTEnts}

1. Introduction $\quad 89$

2. Preliminaries $\quad 92$

3. Uniform bound of the solution 94

$\begin{array}{ll}\text { 4. Stable standing waves } & 95\end{array}$

5. Instability of standing waves $\quad 98$

6. Concluding remarks and open questions 106

$\begin{array}{ll}\text { References } & 110\end{array}$

\section{Introduction}

In this paper, we consider a class of quasilinear Schrödinger equation with a potential $|x|^{2} \varphi$ of the form

(1.1) $\quad i \varphi_{t}=-\triangle \varphi+|x|^{2} \varphi-|\varphi|^{p-1} \varphi-2\left(\triangle|\varphi|^{2}\right) \varphi, \quad t \geq 0, x \in \mathbb{R}^{N}$,

1991 Mathematics Subject Classification. 35J20, 35Q55.

Key words and phrases. Variational methods, standing waves, stability and instability, quasilinear Schrödinger equations. 
where $i^{2}=1, \varphi \equiv \varphi(t, x): \mathbb{R}_{+} \times \mathbb{R}^{N} \rightarrow \mathbb{C}$ is a complex-valued function and $\triangle=$ $\sum_{j=1}^{N} \frac{\partial^{2}}{\partial x_{j}^{2}}$ is the standard Laplacian operator. We are concerned with the stability and the instability of standing waves of (1.1). The main results of the present paper are to prove that the existence of stable standing waves for $1<p<3+\frac{4}{N}$ and study the instability of standing waves for $3+\frac{4}{N} \leq p<2 \cdot 2^{*}-1$, in here and after, $2^{*}=\frac{2 N}{N-2}$ for $N \geq 3$ and $2^{*}=+\infty$ for $N=1,2 ; 2 \cdot 2^{*}=\frac{4 N}{N-2}$ for $N \geq 3$ and $2 \cdot 2^{*}=+\infty$ for $N=1,2$.

Problems of this kind have been derived as models in a lot of physical phenomena. For example, (1.1) models the time evolution of the condensate wave functions in superfluid film [23]. (1.1) has also appeared in plasma physics and fluid mechanics, in the theory of Heisenberg ferromagnet and magnons, and in dissipative quantum mechanics $[20,30,34,37]$.

Compared with the classical semilinear equation with a harmonic potential $|x|^{2} \varphi$ of the form

$$
i \varphi_{t}=-\triangle \varphi+|x|^{2} \varphi-|\varphi|^{p-1} \varphi, t \geq 0, x \in \mathbb{R}^{N},
$$

(1.1) has an additional quasilinear term $\left(\triangle|\varphi|^{2}\right) \varphi$. This term is physically relevant but creates great difficulties in mathematical studies. For example, given an initial condition

$$
\left.\varphi(t, x)\right|_{t=0}=\varphi_{0}(x),
$$

it is proved that the local solution of Eqs. $(1.2)+(1.3)$ exists for any initial value $\varphi_{0}$ in the energy space $W^{1,2}\left(\mathbb{R}^{N}\right)$ and $1<p<2^{*}-1$, see e.g. [6]. But for Cauchy problem $(1.1)+(1.3)$, it is still unknown if the local solution exists for any initial value $\varphi_{0} \in W^{1,2}\left(\mathbb{R}^{N}\right)$. Only when $\varphi_{0}$ is sufficiently smooth, Poppenberg [28] has proved the existence of local solutions of $(1.1)+(1.3)$. For some other results on the related Cauchy problems, we refer the interested readers to Kenig et al. [22] and de Bouard et al. [3]. It is also known from [6] that, when $1<p<1+\frac{4}{N}$, the solution of Eqs.(1.2)+(1.3) exists globally in time for any $\varphi_{0} \in W^{1,2}\left(\mathbb{R}^{N}\right)$; and when $1+\frac{4}{N} \leq p<2^{*}-1$, solutions of Eqs.(1.2)+(1.3) may blow up in finite time for suitable initial value $\varphi_{0}$, see e.g. $[\mathbf{6}, \mathbf{1 5}]$. While for Eqs. $(1.1)+(1.3)$, it is proved that, when $3+\frac{4}{N} \leq p<2 \cdot 2^{*}-1$, the solution of Eqs.(1.1) $+(1.3)$ may blow up in a finite time for suitable $\varphi_{0}$, see $[\mathbf{1 9}]$. Moreover the existence of global solutions of Eqs. (1.1)+(1.3) was an open problem for a long time. The main difficulty is due to the presence of the quasilinear term $\left(\triangle|\varphi|^{2}\right) \varphi$.

In the present paper, we do not discuss the Cauchy problem of (1.1). We are interested in the existence of stable and unstable standing waves of (1.1), which is an important and interesting issue of nonlinear Schrödinger equations. Throughout this paper, we make the following assumption:

Assumption (A): Suppose that $k \geq \frac{N}{2}+7$ and $\varphi_{0} \in W^{k, 2}\left(\mathbb{R}^{N}\right) \cap L^{2}\left(\mathbb{R}^{N},|x|^{2} d x\right)$. There is a maximal $T>0$ and a unique solution $\varphi(t, x)$ of Eqs.(1.1) $+(1.3)$ such that $\varphi \in C\left([0, T), W^{k, 2}\left(\mathbb{R}^{N}\right) \cap L^{2}\left(\mathbb{R}^{N},|x|^{2} d x\right)\right)$, and for all $t \in[0, T)$ there hold $\int|\varphi(t, \cdot)|^{2}=\int\left|\varphi_{0}\right|^{2}$, and $E(\varphi(t, \cdot))=E\left(\varphi_{0}\right)$, where

$$
E(\psi)=\int \frac{1}{2}\left(|\nabla \psi|^{2}+|x|^{2}|\psi|^{2}+\left.\left.|\nabla| \psi\right|^{2}\right|^{2}\right)-\frac{1}{p+1} \int|\psi|^{p+1}
$$

for any $\psi \in W^{k, 2}\left(\mathbb{R}^{N}\right) \cap L^{2}\left(\mathbb{R}^{N},|x|^{2} d x\right)$. 
We will prove the existence of stable standing waves for $1<p<3+\frac{4}{N}$ and study the instability of standing waves for $3+\frac{4}{N} \leq p<2 \cdot 2^{*}-1$. Firstly, we recall some results on the standing waves of (1.2). By a standing wave, we mean a special periodic solution of the form $e^{i \mu t} u(x)$, where $\mu \in \mathbb{R}$ and $u \equiv u(x)$ is a minimal action solution of the following elliptic equation

$$
-\triangle u+\mu u+|x|^{2} u=|u|^{p-1} u, \quad u \rightarrow 0(|x| \rightarrow \infty) .
$$

There are many results on the existence of standing waves of (1.2), see [31] and the references therein. When $1<p<1+\frac{4}{N}$, it is proved that standing waves of Eq.(1.2) are orbital stable, see $[\mathbf{7}, \mathbf{3 2}, \mathbf{4 0}]$. When $1+\frac{4}{N}<p<2^{*}-1$, Rose et al. [32] and Fukuizumi [13] proved that there is a sequence $\left\{\mu_{n}\right\}_{n \in \mathbb{N}}$ with $\mu_{n} \rightarrow-\mu_{0}$ as $n \rightarrow \infty$, such that the standing waves $e^{i \mu_{n} t} u(x)$ are orbital stable, where $\mu_{0}$ is the first eigenvalue of the following eigenvalue problem

$$
-\triangle v+|x|^{2} v=\mu v, \quad v \rightarrow 0 \quad \text { as } \quad|x| \rightarrow \infty .
$$

Also when $1+\frac{4}{N}<p<2^{*}-1$, Fukuizumi et al. [13, 14] have proven that there is $\mu^{*}>0$ such that for any $\mu>\mu^{*}$, the standing wave $e^{i \mu t} u(x)$ is orbital unstable. It is also proved that if $\left(N^{2}+4+4 \sqrt{N^{2}+1}\right) / N^{2}<p<2^{*}-1$, then the standing wave $e^{i \mu t} u(x)$ is unstable for all $\mu \in(0,+\infty)$, see [13]. We also refer the interested readers to Shatah et al. [33] and Grillakis et al. $[\mathbf{1 6}, \mathbf{1 7}]$ for related results about the stability and instability of standing waves of semilinear Schrödinger equations without potential of the form $i \varphi_{t}=-\triangle \varphi-|\varphi|^{p-1} \varphi$.

Next we turn to the standing waves of the quasilinear Schrödinger equation (1.1). Again by a standing wave, we mean a special periodic solution of the form $e^{i \omega t} u(x)$, where $\omega \in \mathbb{R}$ and $u \equiv u(x)$ is a minimal action solution of the following elliptic equation

$$
-\triangle u+\omega u+|x|^{2} u-2\left(\triangle|u|^{2}\right) u=|u|^{p-1} u, \quad u \rightarrow 0(|x| \rightarrow \infty) .
$$

Liu et al. [27] and Poppenberg et al. [29] have proved the existence of standing waves of (1.1) for $1<p<2 \cdot 2^{*}-1$. However for the stability of standing waves of (1.1), only a few results are known in the literature. When $N=1$ and without the term $|x|^{2} \varphi$, Iliev et al. [21] have studied the stability of standing waves by using the methods of Grillakis et al. $[\mathbf{1 6}, \mathbf{1 7}]$. Also when $N=1$ and without the term $|x|^{2} \varphi$, the strong instability of standing waves has been obtained in $[\mathbf{9}]$ by the variational methods. While for $N \geq 2$, the existence of stable and unstable standing waves of (1.1) remain open for many years. On the other hand, when $N \geq 2$, Guo et al. [18] have proven that if $1<p<1+\frac{4}{N}$ then a standing wave of $(1.1)$ is orbital stable. But we only have blow-up results of Eqs.(1.1) $+(1.3)$ for $p \geq 3+\frac{4}{N}$, see Guo et al. [19]. So it is reasonable to conjecture that there exist stable standing waves for $1+\frac{4}{N} \leq p<3+\frac{4}{N}$ and there exist unstable standing waves for $p \geq 3+\frac{4}{N}$. For (1.1) with $N \geq 2$ and without potential term $|x|^{2} \varphi$, these phenomena have been completely displayed by the authors in $[\mathbf{1 0}]$. While the existence of stable standing waves for $1<p<3+\frac{4}{N}$ and the existence of unstable standing wave for $3+\frac{4}{N}<p<2 \cdot 2^{*}-1$ was obtained in [12] by different methods. The purpose here is to show these phenomena for (1.1). Our main results are Theorem 4.4 and Theorem 5.11. These results indicate that the quasilinear term $\left(\triangle|\varphi|^{2}\right) \varphi$ makes the standing waves more stable which is consistent with the physical phenomenon. One by-product (see Theorem 3.1) of our results is that, for $p=1+\frac{4}{N}$ and any initial data, the solution of Eqs. $(1.1)+(1.3)$ is uniformly bounded with respect to the time 
of existence, which gives a striking contrast to the blow-up result of Eqs. $(1.2)+(1.3)$ obtained by Zhang [39].

This paper is organized as follows. In Section 2, some preliminaries are given. Especially a variant of Gargliardo-Nirenberg's inequality (see Theorem 2.2) is derived, which will be used conveniently in the study of orbital stability of standing waves of (1.1). In Section 3, we use the variant Gargliardo-Nirenberg's inequality to prove a uniform bound to the solution of Eqs.(1.1)+(1.3). In particular, we show a quite different phenomenon from the results of Zhang [39]. The result obtained in this section implies that the additional quasilinear term allows local solutions to be extended easily to global ones. In Section 4, we prove the existence of an orbital stable standing wave of (1.1) for $N \geq 1$ and $1<p<3+\frac{4}{N}$. The main result is Theorem 4.4. In Section 5, we prove the instability of standing waves of (1.1) for $3+\frac{4}{N} \leq p<2 \cdot 2^{*}-1$ and suitable frequency $\omega$. The main result is contained in Theorem 5.11. In the final section, we give some concluding remarks and some open problems.

Notations. Throughout this paper, $\int \cdot$ always means $\int_{\mathbb{R}^{N}} \cdot d x$. Different positive constants might be denoted by the same letter $C$ or $C_{j} ; L^{q}\left(\mathbb{R}^{N}\right)$ is the usual Lebesgue space with the usual norm $\|\cdot\|_{q} \cdot L^{2}\left(\mathbb{R}^{N},|x|^{2} d x\right)$ is a weighted Lebesgue space. $W^{k, 2}\left(\mathbb{R}^{N}\right)$ (with $k \geq 1$ ) is the standard Sobolev space with the norm $\|u\|_{W^{k, 2}}^{2}$ $=\int\left(|u|^{2}+\sum_{j=1}^{k}\left|\nabla^{j} u\right|^{2}\right) . \nabla^{j} u$ is the $j$ th derivative of $u . H^{k}=\left\{u \in W^{k, 2}\left(\mathbb{R}^{N}\right)\right.$ : $\left.\int|x|^{2}|u|^{2}<+\infty\right\}$. On $H^{1}$, we use the norm $\|u\|_{H^{1}}^{2}=\int\left(|\nabla u|^{2}+|x|^{2}|u|^{2}+|u|^{2}\right)$. Define $X_{k}=\left\{u \in H^{k}:\left.\left.\int|\nabla| u\right|^{2}\right|^{2} d x<+\infty\right\}$. We also use the norm $\|u\|_{H^{1}}^{2}$ on $X_{1}$. $\rightarrow$ denotes strong convergence and $\rightarrow$ denotes weak convergence. Re denotes the real part and Im denotes the imaginary part; and, for any $t \in \mathbb{R}$, the function $x \mapsto \varphi(t, x)$ is simply denoted by $\varphi(t)$, if no confusion occurs.

\section{Preliminaries}

In this section, we give some preliminaries which are useful in what follows. A starting point is the following version of Gagliardo-Nirenberg inequality from [38].

LEMMA 2.1. [38] Let $1<q<2^{*}-1$. There is a positive constant $C$ such that for any $u \in W^{1,2}\left(\mathbb{R}^{N}\right)$,

$$
\int|u|^{q+1} \leq C\left(\int|\nabla u|^{2}\right)^{\frac{N(q-1)}{4}}\left(\int|u|^{2}\right)^{\frac{2(q+1)-N(q-1)}{4}} .
$$

THEOREM 2.2. If $1<p<2 \cdot 2^{*}-1$, then there is a positive constant $C$ such that for any $u \in X_{1}$, there holds

$$
\int|u|^{p+1} \leq C\left(\int|u|^{2}\right)^{\frac{4 N+(p+1)(2-N)}{2 N+4}}\left(\left.\left.\int|\nabla| u\right|^{2}\right|^{2}\right)^{\frac{N(p-1)}{2 N+4}} .
$$

Proof. Firstly, when $3<p<2 \cdot 2^{*}-1$, we choose $q=(p+1) / 2-1$. The choice of $q$ implies that $1<q<2^{*}-1$. Since $u \in X_{1}$, applying Lemma 2.1 to $|u|^{2}$, one gets that

$$
\int|u|^{p+1}=\int|u|^{2(q+1)} \leq C_{1}\left(\int|u|^{4}\right)^{\frac{2(q+1)-N(q-1)}{4}}\left(\left.\left.\int|\nabla| u\right|^{2}\right|^{2}\right)^{\frac{N(q-1)}{4}} .
$$


From the standard interpolation inequality one obtains that

$$
\int|u|^{4} \leq C_{2}\left(\int|u|^{2}\right)^{2 \theta_{1}}\left(\int|u|^{2(q+1)}\right)^{\frac{2\left(1-\theta_{1}\right)}{q+1}},
$$

where $\theta_{1}=\left(\frac{1}{4}-\frac{1}{2(q+1)}\right) /\left(\frac{1}{2}-\frac{1}{2(q+1)}\right)$. Therefore

$$
2 \theta_{1}=\frac{q-1}{q} \quad \text { and } \quad \frac{2\left(1-\theta_{1}\right)}{q+1}=\frac{1}{q} .
$$

Hence, we deduce that

$$
\int|u|^{2(q+1)} \leq C_{3}\left(\int|u|^{2}\right)^{\gamma_{1}}\left(\int|u|^{2(q+1)}\right)^{\gamma_{2}}\left(\left.\left.\int|\nabla| u\right|^{2}\right|^{2}\right)^{\frac{N(q-1)}{4}}
$$

where

$$
\gamma_{1}=\frac{(q-1)(2(q+1)-N(q-1))}{4 q}, \quad \gamma_{2}=\frac{2(q+1)-N(q-1)}{4 q} .
$$

Therefore

$$
\left(\int|u|^{2(q+1)}\right)^{1-\gamma_{2}} \leq C_{3}\left(\int|u|^{2}\right)^{\gamma_{1}}\left(\left.\left.\int|\nabla| u\right|^{2}\right|^{2}\right)^{\frac{N(q-1)}{4}} .
$$

It is now deduced that

$$
\int|u|^{2(q+1)} \leq C\left(\int|u|^{2}\right)^{\gamma_{3}}\left(\left.\left.\int|\nabla| u\right|^{2}\right|^{2}\right)^{\gamma_{4}}
$$

where

$$
\gamma_{3}=\frac{2(q+1)-N(q-1)}{N+2}, \quad \gamma_{4}=\frac{N q}{N+2} .
$$

Recalling that $p+1=2(q+1)$, we get

$$
\int|u|^{p+1} \leq C\left(\int|u|^{2}\right)^{\frac{4 N+(p+1)(2-N)}{2 N+4}}\left(\left.\left.\int|\nabla| u\right|^{2}\right|^{2}\right)^{\frac{N(p-1)}{2 N+4}} .
$$

Secondly, when $1<p \leq 3$, we choose $s$ such that $3<s<2 \cdot 2^{*}-1$ and use the standard interpolation inequality to get that

$$
\|u\|_{p+1}^{p+1} \leq\|u\|_{2}^{(p+1) \theta_{2}}\|u\|_{s+1}^{(p+1)\left(1-\theta_{2}\right)}
$$

where $\theta_{2}=\left(\frac{1}{p+1}-\frac{1}{s+1}\right) /\left(\frac{1}{2}-\frac{1}{s+1}\right)$. Therefore

$$
\|u\|_{p+1}^{p+1} \leq\|u\|_{2}^{\frac{2(s-p)}{s-1}}\|u\|_{s+1}^{\frac{(s+1)(p+1)}{s-1}} .
$$

For $\int|u|^{s+1}$, one obtains from Eq.(2.2) that

$$
\int|u|^{s+1} \leq C\left(\int|u|^{2}\right)^{\frac{4 N+(s+1)(2-N)}{2 N+4}}\left(\left.\left.\int|\nabla| u\right|^{2}\right|^{2}\right)^{\frac{N(s-1)}{2 N+4}} .
$$

Combining (2.4) with (2.3), one has that for $1<p \leq 3$,

$$
\int|u|^{p+1} \leq C\left(\int|u|^{2}\right)^{\frac{4 N+(p+1)(2-N)}{2 N+4}}\left(\left.\left.\int|\nabla| u\right|^{2}\right|^{2}\right)^{\frac{N(p-1)}{2 N+4}}
$$

still holds. The proof is complete. 
Proposition 1. [19] Let $\varphi_{0} \in W^{k, 2}\left(\mathbb{R}^{N}\right)$ with $k \geq \frac{N}{2}+7,|\cdot| \varphi_{0}(\cdot) \in L^{2}\left(\mathbb{R}^{N}\right)$ and $\varphi(t, x)$ be the solution of (1.1) with initial value $\varphi(0, x)=\varphi_{0}(x)$. Denote $D(t)=\int|x|^{2}|\varphi|^{2}$. Then we have that, for all $t \in[0, T)$,

$$
D^{\prime \prime}(t)=8 \int\left(|\nabla \varphi|^{2}-|x|^{2}|\varphi|^{2}+\left.\left.\frac{N+2}{2}|\nabla| \varphi\right|^{2}\right|^{2}-\frac{N(p-1)}{2(p+1)}|\varphi|^{p+1}\right) .
$$

LEMma 2.3. $[\mathbf{1}, \mathbf{3 1}]$ Let $N \geq 1$. The following embedding

$$
H^{1} \hookrightarrow L^{q+1}\left(\mathbb{R}^{N}\right) \text { with } 2 \leq q+1<2^{*},
$$

is compact.

\section{Uniform bound of the solution}

This section is concerned with the uniform bound with respect to the time of existence to the solution of Eqs. (1.1) + (1.3). We point out that for the semilinear equation (1.2), Zhang [39] proved that, when $p=1+\frac{4}{N}$ and $\varphi_{0}(x)=$ $\lambda^{\frac{N}{2}} \Psi(\lambda x)$ (where $\Psi(x)$ is the minimal action solution of $-\Delta u+u=|u|^{\frac{4}{N}} u$ in $\left.W^{1,2}\left(\mathbb{R}^{N}\right)\right)$, the solution of Eqs. $(1.2)+(1.3)$ blows up in a finite time in the sense that $\lim _{t \rightarrow T^{-}}\|\nabla \varphi(t)\|_{2}=\infty$ for some $0<T<+\infty$. While for the quasilinear equations $(1.1)+(1.3)$, we will prove in the following theorem that the additional quasilinear term prevents the blow-up from appearing for any initial data contained in a dense subset of $H^{1}$.

THEOREM 3.1. Let $\varphi_{0} \in H^{k}$ (with $k \geq \frac{N}{2}+7$ ) and $1<p<3+\frac{4}{N}$. If $\varphi(t)$ is a solution of Eqs.(1.1) $+(1.3)$, then $\int\left(|\nabla \varphi(t)|^{2}+|x|^{2}|\varphi(t)|^{2}+\left.\left.|\nabla| \varphi(t)\right|^{2}\right|^{2}\right)$ is uniformly bounded with respect to the time $t$ of existence.

Proof. Since $\varphi(t)$ is a solution of Eqs. $(1.1)+(1.3)$, we obtain from the conserved energy that

$$
E\left(\varphi_{0}\right)=\int \frac{1}{2}\left(|\nabla \varphi(t)|^{2}+|x|^{2}|\varphi(t)|^{2}+\left.\left.|\nabla| \varphi(t)\right|^{2}\right|^{2}\right)-\frac{1}{p+1} \int|\varphi(t)|^{p+1} .
$$

It is now deduced from Theorem 2.2 and Young inequality that

$$
\int\left(|\nabla \varphi(t)|^{2}+|x|^{2}|\varphi(t)|^{2}+\left.\left.|\nabla| \varphi(t)\right|^{2}\right|^{2}\right) \leq 2 E\left(\varphi_{0}\right)+C\left(\int|\varphi(t)|^{2}\right)^{s}
$$

with $s=\frac{4 N+(p+1)(2-N)}{3 N+4-N p}$, which implies that

$$
\int\left(|\nabla \varphi(t)|^{2}+|x|^{2}|\varphi(t)|^{2}+\left.\left.|\nabla| \varphi(t)\right|^{2}\right|^{2}\right)
$$

is uniformly bounded with respect to the existence time $t \in[0, T)$.

Remark. Since $k \geq \frac{N}{2}+7$, the embedding $H^{k} \hookrightarrow C^{1}\left(\mathbb{R}^{N}\right)$ is continuous. Hence, for $u \in H^{k}$, we have that $\left.\left.\int|\nabla| u\right|^{2}\right|^{2}<\infty$. Therefore, we get that for a dense subset of $H^{1}, \int|\nabla \varphi(t)|^{2}$ is always uniformly bounded with respect to the time of existence. 


\section{Stable standing waves}

In this section, we study the existence of an orbital stable standing wave of (1.1). Firstly we need the following definition.

Definition 4.1. A set $S \subset X_{1}$ is said to be $X_{1}$-stable with respect to (1.1) if for any $\varepsilon>0$, there is $\delta>0$ such that for any $\varphi_{0} \in X_{1} \cap H^{k}$ with $k \geq \frac{N}{2}+7$ and

$$
\inf _{v \in S}\left(\left\|\varphi_{0}-v\right\|_{H^{1}}+\left.\left|\int\right| \nabla\left|\varphi_{0}\right|^{2}\right|^{2}-\left.\left.\int|\nabla| v\right|^{2}\right|^{2} \mid\right)<\delta,
$$

the solution $\varphi(t, x) \in C\left([0, T), H^{k}\right)$ of Eqs. $(1.1)+(1.3)$ satisfies

$$
\sup _{0 \leq t<T} \inf _{v \in S}\left(\|\varphi(t, \cdot)-v\|_{H^{1}}+\left.\left|\int\right| \nabla|\varphi(t, \cdot)|^{2}\right|^{2}-\left.\left.\int|\nabla| v\right|^{2}\right|^{2} \mid\right)<\varepsilon .
$$

Otherwise $S$ is called $X_{1}$-unstable. A standing wave $e^{i \omega t} u(x)$ is said to be orbital stable if the set $\left\{e^{i \theta} u(x) ; \theta \in \mathbb{R}\right\}$ is stable.

4.1. Existence of standing waves. In this subsection, we study the existence of a standing wave of (1.1). We point out that Liu et al. $[\mathbf{2 7}, \mathbf{1 1}]$ have got some results on the existence of standing waves of (1.1). However, due to the presence of the quasilinear term, one can not do scaling arguments and hence the results of Liu et al. $[\mathbf{2 7}, \mathbf{1 1}]$ seem to be not suitable for the study of the orbital stability of standing waves of (1.1). Here, we use the method of Cazenave et al. [7]. For any $\Lambda>0$, we consider the following minimization problem

$$
m_{\Lambda}=\inf \left\{F(u): u \in X_{1} \text { and } h(u)=\Lambda\right\},
$$

where $h(u)=\frac{1}{2} \int|u|^{2}$ and

$$
F(u)=\int\left(\frac{1}{2}|\nabla u|^{2}+\frac{1}{2}|x|^{2}|u|^{2}+\left.\left.\frac{1}{2}|\nabla| u\right|^{2}\right|^{2}-\frac{1}{p+1}|u|^{p+1}\right) .
$$

Before solving this minimization problem, we give several lemmas.

LEMMA 4.2. Let $\left\{u_{n}\right\}_{n \in \mathbb{N}} \subset X_{1}$ be a minimizing sequence of $m_{\Lambda}$. If $1<p<$ $3+\frac{4}{N}$, then $\int\left(\left|\nabla u_{n}\right|^{2}+|x|^{2}\left|u_{n}\right|^{2}+\left.\left.|\nabla| u_{n}\right|^{2}\right|^{2}\right)$ is uniformly bounded with respect to $n \in \mathbb{N}$.

Proof. Since $\left\{u_{n}\right\}_{n \in \mathbb{N}} \subset X_{1}$ is a minimizing sequence of $m_{\Lambda}$, for $n$ large enough, one has that

$$
m_{\Lambda}+o(1)=F\left(u_{n}\right) \text { and } \quad h\left(u_{n}\right)=\Lambda .
$$

From $1<p<3+\frac{4}{N}$, Theorem 2.2 and Young inequality, one obtains that

$$
\begin{aligned}
\int\left|u_{n}\right|^{p+1} & \leq\left(\int\left|u_{n}\right|^{2}\right)^{\frac{4 N+(p+1)(2-N)}{2 N+4}}\left(\left.\left.\int|\nabla| u_{n}\right|^{2}\right|^{2}\right)^{\frac{N(p-1)}{2 N+4}} \\
& \leq\left.\left.\frac{1}{4} \int|\nabla| u_{n}\right|^{2}\right|^{2}+C\left\|u_{n}\right\|_{2}^{\beta}
\end{aligned}
$$

where $\beta$ and $C \equiv C(N, p)$ are positive constants whose exact value are not important and do not depend on $n \in \mathbb{N}$. Therefore for $n$ large enough,

$$
m_{\Lambda}+1 \geq \frac{1}{4} \int\left(\left|\nabla u_{n}\right|^{2}+|x|^{2}\left|u_{n}\right|^{2}+\left.\left.|\nabla| u_{n}\right|^{2}\right|^{2}\right)-C(2 \Lambda)^{\frac{\beta}{2}},
$$


which implies that $\int\left(\left|\nabla u_{n}\right|^{2}+|x|^{2}\left|u_{n}\right|^{2}+\left.\left.|\nabla| u_{n}\right|^{2}\right|^{2}\right)$ is uniformly bounded with respect to $n \in \mathbb{N}$.

TheOREM 4.3. Suppose $1<p<3+\frac{4}{N}$. Then for any given $\Lambda>0, m_{\Lambda}$ is achieved at some $u_{0} \neq 0$.

Proof. Let $\left\{u_{n}\right\}_{n \in \mathbb{N}} \subset X_{1}$ be a minimizing sequence of $m_{\Lambda}$. We know from Lemma 4.2 that $\int\left(\left|\nabla u_{n}\right|^{2}+|x|^{2}\left|u_{n}\right|^{2}+\left.\left.|\nabla| u_{n}\right|^{2}\right|^{2}\right)$ is uniformly bounded with respect to $n \in \mathbb{N}$. Since $\int\left|u_{n}\right|^{2}=2 \Lambda$, we know that $\left\{u_{n}\right\}_{n \in \mathbb{N}}$ is bounded in $H^{1}$. Going if necessary to a subsequence, still denoted by $\left\{u_{n}\right\}_{n \in \mathbb{N}}$, we may assume that $u_{n} \rightarrow u_{0}$ in $H^{1}$ and $u_{n} \rightarrow u_{0}$ a.e. in $\mathbb{R}^{N}$. By Lemma 2.3 we have that $u_{n} \rightarrow u_{0}$ strongly in $L^{2}\left(\mathbb{R}^{N}\right)$. Hence $h\left(u_{0}\right)=\Lambda$ and $u_{0} \neq 0$. Moreover from Theorem 2.2, one has that

$$
\int\left|u_{n}\right|^{p+1} \leq\left(\int\left|u_{n}\right|^{2}\right)^{\frac{4 N+(p+1)(2-N)}{2 N+4}}\left(\left.\left.\int|\nabla| u_{n}\right|^{2}\right|^{2}\right)^{\frac{N(p-1)}{2 N+4}} .
$$

Note that $\left.\left.\int|\nabla| u_{n}\right|^{2}\right|^{2}$ is uniformly bounded with respect to $n \in \mathbb{N}$. One obtains that

$$
u_{n} \rightarrow u_{0} \quad \text { strongly in } \quad L^{p+1}\left(\mathbb{R}^{N}\right) \quad \text { for } \quad 1<p<3+\frac{4}{N} .
$$

Next, we claim that as $n \rightarrow \infty$,

$$
\left.\left.\int|\nabla| u_{n}\right|^{2}\right|^{2} \geq\left.\left.\int|\nabla| u_{0}\right|^{2}\right|^{2}
$$

Indeed, when $N \geq 3,\left.\left.\int|\nabla| u_{n}\right|^{2}\right|^{2}$ can be regarded as the $\mathscr{D}^{1,2}\left(\mathbb{R}^{N}\right)$ norm of $L_{n}(x):=$ $\left|u_{n}(x)\right|^{2}$. Since $L_{n}$ is bounded in $\mathscr{D}^{1,2}\left(\mathbb{R}^{N}\right)$, there is $L_{0} \in \mathscr{D}^{1,2}\left(\mathbb{R}^{N}\right)$ such that $L_{n} \rightarrow L_{0}$ in $\mathscr{D}^{1,2}\left(\mathbb{R}^{N}\right)$. Also we may assert that $u_{n} \rightarrow u_{0}$ a.e. in $\mathbb{R}^{N}$ and $L_{n} \rightarrow L_{0}$ a.e. in $\mathbb{R}^{N}$. From this we have $L_{0}=\left|u_{0}\right|^{2}$. Therefore for $n$ large

$$
\left.\left.\int|\nabla| u_{n}\right|^{2}\right|^{2} \geq\left.\left.\int|\nabla| u_{0}\right|^{2}\right|^{2}
$$

When $N=2$, we use the continuous embedding of $W^{1,2}\left(\mathbb{R}^{N}\right)$ into $L^{q}\left(\mathbb{R}^{N}\right)$ for any $2 \leq q<\infty$ (instead of using $\mathscr{D}^{1,2}\left(\mathbb{R}^{N}\right)$ ) and a similar argument works. When $N=1$, the proof of $\int\left|\left(\left|u_{n}\right|^{2}\right)^{\prime}\right|^{2} \geq \int\left|\left(\left|u_{0}\right|^{2}\right)^{\prime}\right|^{2}$ is similar but simpler. Using BrezisLieb Lemma [4], we get that

$$
\begin{aligned}
m_{\Lambda}+o(1)= & \int\left(\frac{1}{2}\left|\nabla u_{n}\right|^{2}+\frac{1}{2}|x|^{2}\left|u_{n}\right|^{2}+\left.\left.\frac{1}{2}|\nabla| u_{n}\right|^{2}\right|^{2}-\frac{1}{p+1}\left|u_{n}\right|^{p+1}\right) \\
\geq & \frac{1}{2} \int\left(\left|\nabla u_{0}\right|^{2}+|x|^{2}\left|u_{0}\right|^{2}+\left.\left.|\nabla| u_{0}\right|^{2}\right|^{2}\right)-\frac{1}{p+1} \int\left|u_{0}\right|^{p+1} \\
& \quad+\frac{1}{2} \liminf _{n \rightarrow \infty} \int\left(\left|\nabla\left(u_{n}-u_{0}\right)\right|^{2}+|x|^{2}\left|u_{n}-u_{0}\right|^{2}\right) \\
\geq & F\left(u_{0}\right) \geq m_{\Lambda} \text { since } h\left(u_{0}\right)=\Lambda .
\end{aligned}
$$

Hence $m_{\Lambda}=F\left(u_{0}\right)$.

Next, we denote by $G_{\Lambda}$ the set of minimizers of $m_{\Lambda}$. We know from Theorem 4.3 that for $\Lambda>0, G_{\Lambda}$ is not empty. Let $u_{0} \in G_{\Lambda}$. Note that for any $\phi \in C_{0}^{\infty}\left(\mathbb{R}^{N}\right)$,

$$
\begin{aligned}
& \operatorname{Re} \int\left(-\triangle\left|u_{0}\right|^{2}\right) u_{0} \bar{\phi} \\
& =\operatorname{Re} \int\left(u_{0}\left|\nabla u_{0}\right|^{2} \bar{\phi}+\bar{u}_{0} \nabla u_{0} \nabla u_{0} \bar{\phi}+u_{0}^{2} \nabla \bar{u}_{0} \nabla \bar{\phi}+\left|u_{0}\right|^{2} \nabla u_{0} \nabla \bar{\phi}\right) .
\end{aligned}
$$


Since

$$
\begin{gathered}
\int\left|u_{0}\right|^{2}\left|\nabla u_{0}\right| \leq \int\left|u_{0}\right|^{2}\left(1+\left|\nabla u_{0}\right|^{2}\right)<+\infty \quad \text { and } \\
\int\left|u_{0}\right|\left|\nabla u_{0}\right|^{2} \leq \int\left|\nabla u_{0}\right|^{2}\left(1+\left|u_{0}\right|^{2}\right)<+\infty
\end{gathered}
$$

we have that $\operatorname{Re} \int \nabla\left(\left|u_{0}\right|^{2}\right) \nabla\left(u_{0} \bar{\phi}\right)$ exists for any $\phi \in C_{0}^{\infty}\left(\mathbb{R}^{N}\right)$. The standard proof of the Ljusternik's Theorem on Lagrange multipliers [5] implies that there exists $\gamma$ such that $u_{0}$ is a weak solution of

$$
-\triangle u+|x|^{2} u-2\left(\triangle|u|^{2}\right) u-|u|^{p-1} u=\gamma u, \quad u \in X_{1} .
$$

Remark 1. We remark that we do not know the $\gamma$ in (4.5) is zero or not since we are facing with a quasilinear term. If $\gamma \neq 0$, then $u(x)$ is a standing wave of (1.1). In Section 6, we will discuss the conditions on $\Lambda, p$ and $N$ such that $\gamma \neq 0$.

4.2. Stability of standing waves. In this subsection, we prove the orbital stability of the standing wave of (1.1) obtained in the above. From the previous subsection, we know that for $\mu=-\gamma, e^{i \mu t} u_{0}(x)$ is a standing wave of (1.1). Hence $G_{\Lambda}\left(u_{0}\right):=\left\{e^{i \mu t} u_{0}(x): t>0\right\}$ is the orbit of $u_{0}$. Moreover, if $v \in G_{\Lambda}\left(u_{0}\right)$ then $e^{i s} v \in G_{\Lambda}\left(u_{0}\right)$ for any $s \in \mathbb{R}$. Then the result of orbital stability is:

TheOREM 4.4. Suppose $1<p<3+\frac{4}{N}$. The set $G_{\Lambda}\left(u_{0}\right)$ is $X_{1}$-stable with respect to (1.1) in the sense of Definition 4.1 .

Proof. Suppose the conclusion is false. Then there exists a number $\varepsilon_{0}>0$, such that for any $m>0$, there is $\varphi_{0 m} \in X_{1} \cap H^{k}$ with $k \geq \frac{N}{2}+7$ such that

$$
\inf _{v \in G_{\Lambda}\left(u_{0}\right)}\left(\left\|\varphi_{0 m}-v\right\|_{H^{1}}+\left.\left|\int\right| \nabla\left|\varphi_{0 m}\right|^{2}\right|^{2}-\left.\left.\int|\nabla| v\right|^{2}\right|^{2} \mid\right)<\frac{1}{m}
$$

and

$$
\sup _{0<t<T_{m}} \inf _{v \in G_{\Lambda}\left(u_{0}\right)}\left(\left\|\varphi_{m}(t, \cdot)-v\right\|_{H^{1}}+\left.\left|\int\right| \nabla\left|\varphi_{m}(t, \cdot)\right|^{2}\right|^{2}-\left.\left.\int|\nabla| v\right|^{2}\right|^{2} \mid\right) \geq \varepsilon_{0},
$$

where $\varphi_{m}(t, \cdot) \in C\left(\left[0, T_{m}\right), H^{k}\right)$ is the solution of $(1.1)$ with initial condition $\varphi_{m}(0, \cdot)$ $=\varphi_{0 m}$. Hence, we can pick the first time $t_{m}$ such that

$$
\inf _{v \in G_{\Lambda}\left(u_{0}\right)}\left(\left\|\varphi_{m}\left(t_{m}, \cdot\right)-v\right\|_{H^{1}}+\left.\left|\int\right| \nabla\left|\varphi_{m}\left(t_{m}, \cdot\right)\right|^{2}\right|^{2}-\left.\left.\int|\nabla| v\right|^{2}\right|^{2} \mid\right)=\varepsilon_{0} .
$$

Since, $\varphi_{0 m}$ converges to some element $g^{*} \in G_{\Lambda}\left(u_{0}\right)$ in the norm $\|\cdot\|_{H^{1}}$ and $m_{\Lambda}=$ $F(g)$ for all $g \in G_{\Lambda}\left(u_{0}\right)$ and $h(g)=\Lambda$, we obtain from (4.6) that $F\left(\varphi_{0 m}\right) \rightarrow m_{\Lambda}$ and $h\left(\varphi_{0 m}\right) \rightarrow \Lambda$ as $m \rightarrow \infty$. Thus we can find a sequence $\beta_{m} \rightarrow 1$ such that $h\left(\beta_{m} \varphi_{0 m}\right)=\Lambda$ for all $m$. Therefore, for $q_{m}=\beta_{m} \varphi_{m}\left(t_{m}, \cdot\right)$, there holds $h\left(q_{m}\right)=\Lambda$. From (4.6) and the conserved energy, we have

$$
\lim _{m \rightarrow \infty} F\left(q_{m}\right)=\lim _{m \rightarrow \infty} F\left(\varphi_{m}\left(t_{m}, \cdot\right)\right)=\lim _{m \rightarrow \infty} F\left(\varphi_{0 m}\right)=m_{\Lambda} .
$$

Therefore $\left\{q_{m}\right\}$ is a minimizing sequence for $m_{\Lambda}$. The proof of Theorem 4.3 implies that $q_{m} \rightarrow q_{0}$ in the norm $\|\cdot\|_{H^{1}}$ and

$$
\left.\left.\lim _{m \rightarrow \infty} \int|\nabla| q_{m}\right|^{2}\right|^{2}=\left.\left.\int|\nabla| q_{0}\right|^{2}\right|^{2} .
$$


Hence $q_{0} \in G_{\Lambda}\left(\left(u_{0}\right)\right)$ and we have that

$$
\begin{aligned}
\varepsilon_{0} \leq & \left\|\varphi_{m}\left(t_{m}, \cdot\right)-q_{0}\right\|_{H^{1}}+\left.\left|\int\right| \nabla\left|\varphi_{m}\left(t_{m}, \cdot\right)\right|^{2}\right|^{2}-\left.\left.\int|\nabla| q_{0}\right|^{2}\right|^{2} \mid \\
= & \left|\frac{1}{\beta_{m}}-1\right|\left\|q_{m}\right\|_{H^{1}}+\left\|q_{m}-q_{0}\right\|_{H^{1}} \\
& +\left.\left.\left|\frac{1}{\beta_{m}^{4}}-1\right| \int|\nabla| q_{m}\right|^{2}\right|^{2}+\left.\left|\int\right| \nabla\left|q_{m}\right|^{2}\right|^{2}-\left.\left.\int|\nabla| q_{0}\right|^{2}\right|^{2} \mid \rightarrow 0
\end{aligned}
$$

as $m \rightarrow \infty$, which is a contradiction.

Remark 2. We point out that the orbital stability of standing waves obtained here is a generalized version of orbital stability of standing waves, see e.g. $[\mathbf{7}, \mathbf{3 6}]$. The main reason is that due to the presence of the quasilinear term $\left(\Delta|u|^{2}\right) u$, we do not know if the minimizer obtained in Theorem 4.3 is unique. We believe that the study of uniqueness of the minimizer is an interesting problem, which will be studied in the future.

Remark 3. Although we have proven that for any given $\Lambda>0$, the standing wave related with a minimizer of the minimization problem $m_{\Lambda}$ is orbital stable by Definition 4.1, we do not know if the nonlinear stability in Theorem 4.4 is true for all ground states with $1<p<3+\frac{4}{N}$, because we can not do scaling argument due to the presence of the quasilinear term $\left(\Delta|u|^{2}\right) u$. We believe that this is an interesting and complicated problem, which can be a problem for further study.

\section{Instability of standing waves}

In this section, we study the instability of standing waves of the quasilinear equation (1.1). For $\omega>0$, we define on $X_{1}$ the following functionals

$$
\begin{gathered}
J(u)=\frac{1}{2} \int\left(|\nabla u|^{2}+\omega|u|^{2}+|x|^{2}|u|^{2}\right)+\left.\left.\frac{1}{2} \int|\nabla| u\right|^{2}\right|^{2}-\frac{1}{p+1} \int|u|^{p+1}, \\
I(u)=\int\left(|\nabla u|^{2}+\omega|u|^{2}+|x|^{2}|u|^{2}\right)+\left.\left.2 \int|\nabla| u\right|^{2}\right|^{2}-\int|u|^{p+1} \text { and } \\
Q(u)=\int\left(|\nabla u|^{2}-|x|^{2}|u|^{2}\right)+\left.\left.\frac{N+2}{2} \int|\nabla| u\right|^{2}\right|^{2}-\frac{N(p-1)}{2(p+1)} \int|u|^{p+1}
\end{gathered}
$$

Remark. Due to the quasilinear term $\left.\left.\int|\nabla| u\right|^{2}\right|^{2}$, the functionals $J, I$ and $Q$ may not be continuous on $X_{1}$.

Define the following Nehari type set

$$
\mathscr{N}_{1}=\left\{u \in X_{1}: I(u)=0 \quad \text { and } u \neq 0\right\}
$$

and its related minimization problem

$$
d_{1}=\inf _{u \in \mathscr{N}_{1}} J(u)
$$

From Liu et al. [27] we know that $d_{1}>0$ and $d_{1}$ is achieved by a $v \in X_{1}$, which is a minimal action solution of the following elliptic equation

$$
-\triangle u+\omega u+|x|^{2} u-2\left(\triangle|u|^{2}\right) u=|u|^{p-1} u, u \rightarrow 0(|x| \rightarrow \infty) .
$$


From an argument similar to [27, Lemma 5.10], one has that $v \in C^{1}\left(\mathbb{R}^{N}\right)$. Moreover, there is $\theta>0$ such that

$$
|v(x)| \leq C_{0} e^{-\theta|x|} \text { and } \int_{\mathbb{R}^{N} \backslash B(0, R)}\left(|\nabla v|^{2}+|v|^{2}\right) \leq C_{0} e^{-\theta R} .
$$

Before studying the instability of standing waves, we introduce another minimization problem. Define

$$
d_{k}=\inf _{u \in \mathscr{N}_{k}} J(u), \quad \text { where } \quad \mathscr{N}_{k}=\left\{u \in X_{k}: I(u)=0 \text { and } u \neq 0\right\} .
$$

The following lemmas are useful in what follows.

LEMMA 5.1. [27] Let $3 \leq p<2 \cdot 2^{*}-1$. If $u \in \mathscr{N}_{k}$, then $J(u) \geq J(s u)$ for any $s>0$ and $s \neq 1$.

LEMMA 5.2. [27] Let $u \neq 0, u \in X_{1}$ and either $p>3$ or $p=3$ and $\left.\left.2 \int|\nabla| u\right|^{2}\right|^{2}<$ $\int|u|^{p+1}$. Then there is a unique $s>0$ such that $s u \in \mathscr{N}_{1}$.

Next, denote $Y:=X_{1} \cap C^{1}\left(\mathbb{R}^{N}\right)$. On $Y$, we introduce a new norm $\|u\|_{Y}=$ $\|u\|_{H^{1}}+\|u\|_{C^{1}\left(\mathbb{R}^{N}\right)}$, where $\|u\|_{C^{1}\left(\mathbb{R}^{N}\right)}=\sup _{0 \leq \alpha \leq 1, x \in \mathbb{R}^{N}}\left|D^{\alpha} u\right|$.

Lemma 5.3. The functionals $J, I$ and $Q$ are continuous on $X_{1} \cap C^{1}\left(\mathbb{R}^{N}\right)$.

Proof. It suffices to prove that the functional

$$
\left.\left.u \in X_{1} \cap C^{1}\left(\mathbb{R}^{N}\right) \mapsto \int|\nabla| u\right|^{2}\right|^{2}
$$

is continuous. Let $\left\{u_{n}\right\}_{n \in \mathbb{N}} \subset X_{1} \cap C^{1}\left(\mathbb{R}^{N}\right)$ and $u_{n} \rightarrow u$ in $X_{1} \cap C^{1}\left(\mathbb{R}^{N}\right)$, that is to say,

$$
\left\|u_{n}-u\right\|_{H^{1}} \rightarrow 0 \quad \text { and } \quad \sup _{0 \leq \alpha \leq 1, x \in \mathbb{R}^{N}}\left|D^{\alpha} u_{n}-D^{\alpha} u\right| \rightarrow 0 \quad(n \rightarrow \infty) .
$$

We denote $u_{n}(x)=a_{n}(x)+i b_{n}(x)$ and $u(x)=a(x)+i b(x)$, where $a_{n}(x), b_{n}(x)$, $a(x)$ and $b(x)$ are all real-valued functions. Moreover, we have

$$
\left\|a_{n}-a\right\|_{H^{1}} \rightarrow 0, \quad \sup _{0 \leq \alpha \leq 1, x \in \mathbb{R}^{N}}\left|D^{\alpha} a_{n}-D^{\alpha} a\right| \rightarrow 0
$$

and

$$
\left\|b_{n}-b\right\|_{H^{1}} \rightarrow 0, \quad \sup _{0 \leq \alpha \leq 1, x \in \mathbb{R}^{N}}\left|D^{\alpha} b_{n}-D^{\alpha} b\right| \rightarrow 0 .
$$

Observe that

$$
\left.\left.\int|\nabla| u_{n}\right|^{2}\right|^{2}=4 \int a_{n}^{2}\left|\nabla a_{n}\right|^{2}+8 \int a_{n} b_{n} \nabla a_{n} \nabla b_{n}+4 \int b_{n}^{2}\left|\nabla b_{n}\right|^{2} .
$$

In the first place, we have

$$
\left.\left|\int a_{n}^{2}\right| \nabla a_{n}\right|^{2}-\int a^{2}|\nabla a|^{2}\left|\leq \int\right| a_{n}^{2}-\left.a^{2}|| \nabla a_{n}\right|^{2}+\left.\int a^{2}|| \nabla a_{n}\right|^{2}-|\nabla a|^{2} \mid .
$$

Since $\int\left|\nabla a_{n}\right|^{2}$ is uniformly bounded with respect to $n$ and

$$
\sup _{0 \leq \alpha \leq 1, x \in \mathbb{R}^{N}}\left|D^{\alpha} a_{n}-D^{\alpha} a\right| \rightarrow 0,
$$

we have that

$$
\int\left|a_{n}^{2}-a^{2}\right|\left|\nabla a_{n}\right|^{2} \rightarrow 0 \quad \text { as } \quad n \rightarrow+\infty
$$


From $a \in X_{1} \cap C^{1}\left(\mathbb{R}^{N}\right)$ and $\left\|a_{n}-a\right\|_{H^{1}} \rightarrow 0$, one gets that

$$
\left.\int a^{2}|| \nabla a_{n}\right|^{2}-|\nabla a|^{2} \mid \rightarrow 0 \quad \text { as } n \rightarrow+\infty \text {. }
$$

Therefore $\int a_{n}^{2}\left|\nabla a_{n}\right|^{2} \rightarrow \int a^{2}|\nabla a|^{2}$.

Similarly, one can prove that

$$
\int b_{n}^{2}\left|\nabla b_{n}\right|^{2} \rightarrow \int b^{2}|\nabla b|^{2} \quad \text { and } \quad \int a_{n} b_{n} \nabla a_{n} \nabla b_{n} \rightarrow \int a b \nabla a \nabla b .
$$

Hence $\left.\left.\left.\left.\int|\nabla| u_{n}\right|^{2}\right|^{2} \rightarrow \int|\nabla| u\right|^{2}\right|^{2}$. The proof is complete.

Lemma 5.4. Let $3 \leq p<2 \cdot 2^{*}-1$. Then $d_{1}=d_{k}$ for any $k \geq \frac{N}{2}+7$.

Proof. From the definition of $d_{1}$ and $d_{k}$, we know that $d_{k} \geq d_{1}$. Hence it suffices to prove that $d_{1} \geq d_{k}$ (with $k \geq \frac{N}{2}+7$ ). Since $d_{1}$ is achieved by $v \in X_{1}$, one only needs to prove that for any $\varepsilon>0$,

$$
J(v) \geq \inf _{u \in \mathscr{N}_{k}} J(u)-\varepsilon .
$$

The proof of (5.4) is divided into several steps.

Step 1. Constructing a sequence of functions in $H^{k}$ by the properties of $v$.

In fact from $v \in C^{1}$, we have $v \in Y:=X_{1} \cap C^{1}\left(\mathbb{R}^{N}\right)$. Since $H^{k}\left(\mathbb{R}^{N}\right)$ is dense in $H^{1}\left(\mathbb{R}^{N}\right)$ and $H^{k}\left(\mathbb{R}^{N}\right) \hookrightarrow C^{1}\left(\mathbb{R}^{N}\right)$ for $k \geq \frac{N}{2}+7$, we have a sequence $\psi_{n} \in H^{k}$ such that

$$
\psi_{n} \rightarrow v \text { in } H^{1} \text { and } \psi_{n} \rightarrow v \text { in } C^{1} .
$$

Step 2. From the choice of $\psi_{n}$, it is easy to see that

$$
\lim _{n \rightarrow \infty} \int\left(\left|\nabla \psi_{n}-\nabla v\right|^{2}+|x|^{2}\left|\psi_{n}-v\right|^{2}+\omega\left|\psi_{n}-v\right|^{2}\right)=0 .
$$

Step 3. We claim that

$$
\left.\left.\lim _{n \rightarrow \infty} \int|\nabla| \psi_{n}\right|^{2}\right|^{2}=\left.\left.\int|\nabla| v\right|^{2}\right|^{2}
$$

Indeed, note that

$$
\begin{aligned}
& \lim _{n \rightarrow \infty} \int\left(\left.\left.|\nabla| \psi_{n}\right|^{2}\right|^{2}-\left.\left.|\nabla| v\right|^{2}\right|^{2}\right) \\
& \leq\left. 4 \lim _{n \rightarrow \infty} \int|| \psi_{n}\right|^{2}-\left.|v|^{2}|| \nabla \psi_{n}\right|^{2}+\left.4 \lim _{n \rightarrow \infty} \int|v|^{2}|| \nabla \psi_{n}\right|^{2}-|\nabla v|^{2} \mid \\
& \leq 4 \lim _{n \rightarrow \infty}\left(\left.\sup _{x \in \mathbb{R}^{N}}|| \psi_{n}\right|^{2}-\left.|v|^{2}\left|\int\right| \nabla \psi_{n}\right|^{2}\right) \\
& +\left.4 \sup _{x \in \mathbb{R}^{N}}|v|^{2} \lim _{n \rightarrow \infty} \int|| \nabla \psi_{n}\right|^{2}-|\nabla v|^{2} \mid .
\end{aligned}
$$

From $\psi_{n} \rightarrow v$ in $X_{1} \cap C^{1}$, one obtains that

$$
\left.\lim _{n \rightarrow \infty}\left|\int\right| \nabla\left|\psi_{n}\right|^{2}\right|^{2}-\left.\left.\int|\nabla| v\right|^{2}\right|^{2} \mid=0
$$

The proof of Step 3 is complete.

Step 4. There is a sequence $\lambda_{n} \in(0,+\infty)$ such that $\lambda_{n} \psi_{n} \in \mathscr{N}_{k}$ and $\lim _{n \rightarrow \infty} \lambda_{n}=1$. 
Note that $v$ is a solution of (5.2) and

$$
\int\left(|\nabla v|^{2}+\omega|v|^{2}+|x|^{2}|v|^{2}\right)+\left.\left.2 \int|\nabla| v\right|^{2}\right|^{2}=\int|v|^{p+1} .
$$

We obtain from Step 2 and Step 3 that for $n$ large enough,

$$
\left.\left.2 \int|\nabla| \psi_{n}\right|^{2}\right|^{2}<\int\left|\psi_{n}\right|^{p+1}
$$

Hence, the existence of $\lambda_{n}$ follows directly from Lemma 5.1 and Lemma 5.2. It remains to prove that $\lim _{n \rightarrow \infty} \lambda_{n}=1$.

Firstly, we claim that there is $\lambda^{\prime}>0$ such that $\lambda_{n} \geq \lambda^{\prime}>0$ for $n$ large enough. Indeed, if this is not the case (that is to say, $\lambda_{n}>0$ and $\lambda_{n} \rightarrow 0$ as $n \rightarrow \infty$ ), we have from $\lambda_{n} \psi_{n} \in \mathscr{N}_{k}$ that

$$
\int\left(\left|\nabla \psi_{n}\right|^{2}+|x|^{2}\left|\psi_{n}\right|^{2}+\omega\left|\psi_{n}\right|^{2}+\left.\left.2 \lambda_{n}^{2}|\nabla| \psi_{n}\right|^{2}\right|^{2}\right)=\int \lambda_{n}^{p-1}\left|\psi_{n}\right|^{p+1}
$$

Therefore

$$
\lim _{n \rightarrow \infty} \int\left(\left|\nabla \psi_{n}\right|^{2}+|x|^{2}\left|\psi_{n}\right|^{2}+\omega\left|\psi_{n}\right|^{2}\right)=0,
$$

which contradicts the result claimed in Step 2 and $v \neq 0$.

Similarly, we can show that there is $\lambda^{\prime \prime}<+\infty$ such that $\lambda_{n} \leq \lambda^{\prime \prime}<+\infty$ for $n$ large enough. Going if necessary to a subsequence, still denoted by $\left\{\lambda_{n}\right\}_{n \in \mathbb{N}}$, we may assume that

$$
\lim _{n \rightarrow \infty} \lambda_{n}=\lambda_{0} \neq 0
$$

From the previous proof we have that $\lambda_{0} v \in \mathscr{N}_{1}$. Since $v \in \mathscr{N}_{1}$, Lemma 5.1 and Lemma 5.2 imply that $\lambda_{0}=1$. This completes the proof of Step 4 .

Step 5. The proof of (5.4).

From Step 2, Step 3 and Step 4, we know that there is $n_{1}>0$ such that for $n>n_{1}$

and

$$
\begin{aligned}
& \frac{1}{2} \int\left(|\nabla v|^{2}+|x|^{2}|v|^{2}+\omega|v|^{2}\right) \\
& \geq \frac{1}{2} \int\left(\left|\nabla \psi_{n}\right|^{2}+|x|^{2}\left|\psi_{n}\right|^{2}+\omega\left|\psi_{n}\right|^{2}\right)-\frac{\varepsilon}{6} \\
& \geq \frac{1}{2} \int\left(\left|\nabla\left(\lambda_{n} \psi_{n}\right)\right|^{2}+|x|^{2}\left|\lambda_{n} \psi_{n}\right|^{2}+\omega\left|\lambda_{n} \psi_{n}\right|^{2}\right)-\frac{\varepsilon}{3}
\end{aligned}
$$

$$
\left.\left.\frac{1}{2} \int|\nabla| v\right|^{2}\right|^{2} \geq\left.\left.\frac{1}{2} \int|\nabla| \psi_{n}\right|^{2}\right|^{2}-\frac{\varepsilon}{6} \geq\left.\left.\frac{1}{2} \int|\nabla| \lambda_{n} \psi_{n}\right|^{2}\right|^{2}-\frac{\varepsilon}{3} .
$$

Using Theorem 2.2 and Step 4, one has $n_{2}>0$ such that, for $n>n_{2}$,

$$
-\frac{1}{p+1} \int|v|^{p+1} \geq-\frac{1}{p+1} \int\left|\lambda_{n} \psi_{n}\right|^{p+1}-\frac{\varepsilon}{3} \text {. }
$$

Therefore, three exists $n_{0}>\max \left\{n_{1}, n_{2}\right\}$ such that

$$
J(v) \geq J\left(\lambda_{n_{0}} \psi_{n_{0}}\right)-\varepsilon .
$$

Thus (5.4) holds since $\lambda_{n_{0}} \psi_{n_{0}} \in \mathscr{N}_{k}$. The proof is complete.

LemMA 5.5. Let $v$ be a minimizer of (5.1). Then $Q(v)=0$. 
Proof. The idea is based on Pohozaev type identities. Define $\chi_{0} \in C_{0}^{\infty}(\mathbb{R})$ such that $0 \leq \chi_{0} \leq 1$ and $\chi_{0}(s)=1$ if $0 \leq|s| \leq 1, \chi_{0}(s)=0$ if $|s| \geq 2$. We set $\chi_{n}(x)=\chi_{0}\left(\frac{|x|^{2}}{n^{2}}\right), n \in \mathbb{N}$.

Noting firstly that $v$ is a minimizer of problem (5.1) with value $d_{1}$, one has that

$$
\int\left(|\nabla v|^{2}+|x|^{2}|v|^{2}+\omega|v|^{2}+\left.\left.2|\nabla| v\right|^{2}\right|^{2}-|v|^{p+1}\right)=0
$$

Next, multiplying the following equation

$$
-\omega v-|x|^{2} v+\triangle v+2\left(\triangle|v|^{2}\right) v+|v|^{p-1} v=0
$$

by $x \nabla\left(\chi_{n}(x) v\right)$ and integrating over $\mathbb{R}^{N}$, one obtains that

$$
\int x \nabla\left(\chi_{n}(x) v\right)\left(-\omega v-|x|^{2}|v|^{2}+\triangle v+2\left(\triangle|v|^{2}\right) v+|v|^{p-1} v\right)=0 .
$$

Note that $x \nabla\left(\chi_{n}(x) v\right)=\frac{2}{n^{2}}|x|^{2} v \chi_{n}^{\prime}\left(\frac{|x|^{2}}{n^{2}}\right)+\chi_{n}(x) x \nabla v$. It is deduced from integrating by parts that

$$
\begin{aligned}
\int x \nabla\left(\chi_{n}(x) v\right)(-\omega v) & =-\omega \int x|v|^{2} \nabla\left(\chi_{n}(x)\right)-\omega \int v x \nabla v \\
& =-\omega \int \frac{2|x|^{2}}{n^{2}}|v|^{2} \chi_{n}^{\prime}\left(\frac{|x|^{2}}{n^{2}}\right)+\frac{N}{2} \omega \int|v|^{2} .
\end{aligned}
$$

Using Lebesgue dominated convergence theorem, one gets that

$$
\int x \nabla\left(\chi_{n}(x) v\right)(-\omega v) \rightarrow \frac{N}{2} \omega \int|v|^{2} \quad \text { as } \quad n \rightarrow \infty .
$$

Since

and

$$
\begin{aligned}
\int x \nabla\left(\chi_{n}(x) v\right) \triangle v & =\int \triangle v\left(x v \nabla\left(\chi_{n}(x)\right)+\chi_{n}(x) x \nabla v\right) \\
& =\int x v \triangle v \nabla\left(\chi_{n}(x)\right)+\int x_{n}(x) \triangle v \nabla v \\
\int x \chi_{n}(x) \triangle v \nabla v & =-\int \nabla v \nabla\left(x \chi_{n}(x) \nabla v\right) \\
& =-\int \nabla v \nabla\left(\chi_{n}(x)\right)(x \nabla v)-\int \chi_{n}(x) \nabla v \nabla(x \nabla v),
\end{aligned}
$$

$$
\begin{aligned}
& -\int \chi_{n}(x) \nabla v \nabla(x \nabla v)=-\int \chi_{n}(x)|\nabla v|^{2}-\frac{1}{2} \int \chi_{n}(x) x \nabla|\nabla v|^{2} \\
& =-\int \chi_{n}(x)|\nabla v|^{2}+\int x|\nabla v|^{2} \nabla\left(\chi_{n}(x)\right)+\frac{N}{2} \int \chi_{n}(x)|\nabla v|^{2} \\
& =\left(\frac{N}{2}-1\right) \int \chi_{n}(x)|\nabla v|^{2}+\int x|\nabla v|^{2} \nabla\left(\chi_{n}(x)\right),
\end{aligned}
$$

using again Lebesgue dominated convergence theorem, one deduces that

$$
\int x \nabla\left(\chi_{n}(x) v\right) \Delta v \rightarrow\left(\frac{N}{2}-1\right) \int|\nabla v|^{2} \quad \text { as } \quad n \rightarrow \infty .
$$

Similarly one can obtain that as $n \rightarrow \infty$

$$
\int|v|^{p-1} v x \nabla\left(\chi_{n}(x) v\right) \rightarrow-\frac{N}{p+1} \int|v|^{p+1}
$$




$$
\left.\left.\int 2\left(\triangle|v|^{2}\right) v x \nabla\left(\chi_{n}(x) v\right) \rightarrow\left(\frac{N}{2}-1\right) \int|\nabla| v\right|^{2}\right|^{2}
$$

and

$$
\int|x|^{2} v x \nabla\left(\chi_{n}(x) v\right) \rightarrow-\frac{N+2}{2} \int|x|^{2}|v|^{2}
$$

Therefore

$$
\begin{aligned}
\frac{N}{2} \int \omega|v|^{2}+ & \left.\frac{N}{2}-1\right) \int|\nabla v|^{2}+\frac{N+2}{2} \int|x|^{2}|v|^{2}-\frac{N}{p+1} \int|v|^{p+1} \\
& +\left.\left.\left(\frac{N}{2}-1\right) \int|\nabla| v\right|^{2}\right|^{2}=0 .
\end{aligned}
$$

Combining (5.6) with (5.13), one gets that

$$
Q(v)=\int\left(|\nabla v|^{2}-|x|^{2}|v|^{2}+\left.\left.\frac{N+2}{2}|\nabla| v\right|^{2}\right|^{2}-\frac{N(p-1)}{2(p+1)}|v|^{p+1}\right)=0 .
$$

The proof is complete.

Definition 5.6. Let $v$ be a minimal action solution of (5.2). For $\varepsilon>0$, we define a tabular neighborhood around the orbit $\left\{e^{i s} v(x): s \in \mathbb{R}\right\}$ by

$$
U_{\varepsilon}^{(1)}(v)=\left\{\xi \in X_{1} ; \quad \inf _{s \in \mathbb{R}}\left(\left\|\xi-e^{i s} v\right\|_{H^{1}}+\left.\left|\int\right| \nabla|\xi|^{2}\right|^{2}-\left.\left.\int|\nabla| v\right|^{2}\right|^{2} \mid\right)<\varepsilon\right\} .
$$

We also define

$$
U_{\varepsilon}^{(k)}(v)=\left\{\xi \in X_{k} ; \quad \inf _{s \in \mathbb{R}}\left(\left\|\xi-e^{i s} v\right\|_{H^{1}}+\left.\left|\int\right| \nabla|\xi|^{2}\right|^{2}-\left.\left.\int|\nabla| v\right|^{2}\right|^{2} \mid\right)<\varepsilon\right\} .
$$

LEMmA 5.7. There exist $\varepsilon_{0}>0$ and $\sigma_{0}>0$ such that, for any $\xi \in U_{\varepsilon_{0}}^{(1)}(v) \cap$ $C^{1}\left(\mathbb{R}^{N}\right)$ and $\lambda \equiv \lambda(\xi) \in\left(1-\sigma_{0}, 1+\sigma_{0}\right)$, it holds

$$
I\left(\xi^{\lambda}\right)=0,
$$

where we have used the notation $u^{\mu}(x)=\mu^{\frac{N}{2}} u(\mu x)$ for $\mu>0$.

Proof. The proof is based on an application of the implicit function theorem. In the first place, since $I(v)=0$, one obtains that

$$
\left.I\left(\xi^{\lambda}\right)\right|_{\lambda=1, \xi=v}=I(v)=0 .
$$

In the second place, one has

$$
\begin{gathered}
\left.\frac{\partial}{\partial \lambda} I\left(\xi^{\lambda}\right)\right|_{\lambda=1, \xi=v}=2 \int\left(|\nabla v|^{2}-|x|^{2}|v|^{2}\right)+\left.\left.2(N+2) \int|\nabla| v\right|^{2}\right|^{2} \\
-\frac{N}{2}(p-1) \int|v|^{p+1}
\end{gathered}
$$

From $Q(v)=0$, we get that

$$
\begin{aligned}
\left.\frac{\partial}{\partial \lambda} I\left(\xi^{\lambda}\right)\right|_{\lambda=1, \xi=v}=(N & +2)\left.\left.\int|\nabla| v\right|^{2}\right|^{2} \\
& +\left(\frac{N(p-1)}{p+1}-\frac{N}{2}(p-1)\right) \int|v|^{p+1} .
\end{aligned}
$$


It is now deduced from $I(v)=0$ that

$$
\begin{aligned}
\left.\frac{\partial}{\partial \lambda} I\left(\xi^{\lambda}\right)\right|_{\lambda=1, \xi=v}=-\int & \left(|\nabla v|^{2}+\omega|v|^{2}+|x|^{2}|v|^{2}\right) \\
& +\left(\frac{N(p-1)}{p+1}-\frac{N(p-1)}{2}+N+2\right) \int|v|^{p+1} .
\end{aligned}
$$

Since $p \geq 3+\frac{4}{N}$, we have that

$$
\frac{N(p-1)}{p+1}-\frac{N(p-1)}{2}+N+2 \leq 0 .
$$

Therefore

$$
\left.\frac{\partial}{\partial \lambda} I\left(\xi^{\lambda}\right)\right|_{\lambda=1, \xi=v}<0 .
$$

Combining these with the implicit function theorem, we know that Lemma 5.7 holds.

LEMMA 5.8. Let $v$ be the minimal action solution of (5.2) mentioned above. If

$$
\left.\frac{\partial^{2}}{\partial \lambda^{2}} E\left(v^{\lambda}\right)\right|_{\lambda=1}<0
$$

then there exist $\varepsilon_{1}>0$ and $\sigma_{1}>0$ such that for any $\xi \in U_{\varepsilon_{1}}^{(1)}(v) \cap C^{1}\left(\mathbb{R}^{N}\right)$ satisfying $\|\xi\|_{2}=\|v\|_{2}$, there exists $\lambda \in\left(1-\sigma_{1}, 1+\sigma_{1}\right)$ such that

$$
E(v)<E(\xi)+(\lambda-1) Q(\xi)
$$

Proof. Since $\left.\frac{\partial^{2}}{\partial \lambda^{2}} E\left(v^{\lambda}\right)\right|_{\lambda=1}<0$, there exist $\varepsilon_{1}>0$ and $\sigma_{1}>0$ such that

$$
\frac{\partial^{2}}{\partial \lambda^{2}} E\left(\xi^{\lambda}\right)<0
$$

holds for any $\lambda \in\left(1-\sigma_{1}, 1+\sigma_{1}\right)$ and $\xi \in U_{\varepsilon_{1}}^{(1)}(v) \cap C^{1}\left(\mathbb{R}^{N}\right)$. After direct calculations, we have that

$$
\left.\frac{\partial}{\partial \lambda} E\left(\xi^{\lambda}\right)\right|_{\lambda=1}=Q(\xi)
$$

At the same time, the Taylor expansion of $E\left(\xi^{\lambda}\right)$ at $\lambda=1$ is

$$
E\left(\xi^{\lambda}\right)=E(\xi)+Q(\xi)(\lambda-1)+\left.\frac{1}{2} \frac{\partial^{2}}{\partial \lambda^{2}} E\left(\xi^{\lambda}\right)\right|_{\lambda=\tilde{\lambda}}(\lambda-1)^{2}
$$

for some $\tilde{\lambda} \in\left(1-\sigma_{1}, 1+\sigma_{1}\right)$. From lemma 5.7, we can take $0<\varepsilon_{1}<\varepsilon_{0}$ and $0<$ $\sigma_{1}<\sigma_{0}$ such that $I\left(\xi^{\lambda}\right)=0$ for any $\xi \in U_{\varepsilon_{1}}^{(1)}(v) \cap C^{1}\left(\mathbb{R}^{N}\right)$ and $\lambda \in\left(1-\sigma_{1}, 1+\sigma_{1}\right)$. On the other hand, for any $\xi \in U_{\varepsilon_{1}}^{(1)}(v) \cap C^{1}\left(\mathbb{R}^{N}\right)$ satisfying $\|\xi\|_{2}=\|v\|_{2}$, one has that

$$
\left\|\xi^{\lambda}\right\|_{2}=\|\xi\|_{2}=\|v\|_{2}
$$

Therefore, one obtains that

$$
E\left(\xi^{\lambda}\right)=J\left(\xi^{\lambda}\right)-\frac{\omega}{2} \int\left|\xi^{\lambda}\right|^{2} \geq J(v)-\frac{\omega}{2} \int|v|^{2}=E(v) .
$$

Combining (5.15) with (5.16), we get the conclusion of Lemma 5.8. 
DEFINITION 5.9. Let $v$ be the minimal action solution of (5.2) mentioned above. For any $\varphi_{0} \in U_{\varepsilon_{1}}^{(k)}(v)$, we define the maximal time of existence in $U_{\varepsilon_{1}}^{(k)}(v)$ as follows

$$
T\left(\varphi_{0}\right)=\sup \left\{T>0: \varphi(t) \in U_{\varepsilon_{1}}^{(k)}(v) \text { and } 0 \leq t<T\right\},
$$

where $\varphi(t)$ is the solution of (1.1) with initial value $\varphi_{0}$.

Define the set

$$
\Pi=\left\{\xi \in U_{\varepsilon_{1}}^{(1)}(v): E(\xi)<E(v),\|\xi\|_{2}=\|v\|_{2}, \text { and } Q(\xi)<0\right\} .
$$

LEMMA 5.10. Let $v$ be the minimal action solution of (5.2). If

$$
\left.\frac{\partial^{2}}{\partial \lambda^{2}} E\left(v^{\lambda}\right)\right|_{\lambda=1}<0,
$$

then for any $\varphi_{0} \in \Pi \cap H^{k}$, there exists $\sigma_{3}>0$ such that the solution $\varphi(t)$ of (1.1) with initial value $\varphi_{0}$ satisfies

$$
Q(\varphi(t))<-\sigma_{3} \quad \text { for } \quad 0 \leq t<T\left(\varphi_{0}\right) .
$$

Proof. Since $\varphi_{0} \in \Pi \cap H^{k}, \varphi_{0}$ satisfies $E\left(\varphi_{0}\right)<E(v),\left\|\varphi_{0}\right\|_{2}=\|v\|_{2}, Q\left(\varphi_{0}\right)<0$. Put $\sigma_{2}=E(v)-E\left(\varphi_{0}\right)$. Since $\|\varphi(t)\|_{2}=\left\|\varphi_{0}\right\|_{2}, \varphi(t) \in H^{k}\left(\mathbb{R}^{N}\right)$ for any $0 \leq t<$ $T\left(\varphi_{0}\right)$ and for $k \geq \frac{N}{2}+7$,

is continuous, we obtain that

$$
H^{k}\left(\mathbb{R}^{N}\right) \hookrightarrow C^{1}\left(\mathbb{R}^{N}\right)
$$

$$
\varphi(t) \in \Pi \cap C^{1}\left(\mathbb{R}^{N}\right)
$$

for $0 \leq t<T\left(\varphi_{0}\right)$ by the definition of $T\left(\varphi_{0}\right)$. It is deduced from Lemma 5.8 that

$$
Q(\varphi(t))(\lambda-1)+E(\varphi(t))>E(v)
$$

for any $\lambda \in\left(1-\sigma_{1}, 1+\sigma_{1}\right)$ and $0 \leq t<T\left(\varphi_{0}\right)$. Then, by the identity of energy conservation $E(\varphi(t))=E\left(\varphi_{0}\right)$, we get that

$$
Q(\varphi(t))(\lambda-1)>E(v)-E(\varphi(t))=E(v)-E\left(\varphi_{0}\right)=\sigma_{2}>0 .
$$

Thus

$$
Q(\varphi(t)) \neq 0
$$

for any $\lambda \in\left(1-\sigma_{1}, 1+\sigma_{1}\right)$ and $0 \leq t<T\left(\varphi_{0}\right)$. Since $\varphi(t) \in H^{k}$ for all $0 \leq t<T\left(\varphi_{0}\right)$ and $H^{k} \hookrightarrow C^{1}\left(\mathbb{R}^{N}\right)$ is continuous, we obtain from Lemma 5.3 that $Q(\varphi(t))$ is continuous with respect to $t$. At the same time noting the fact that $Q\left(\varphi_{0}\right)<0$, we have that $Q(\varphi(t))<0$ for any $0 \leq t<T\left(\varphi_{0}\right)$. Since $\lambda-1 \geq-\sigma_{1}$ for any $0 \leq t<T\left(\varphi_{0}\right)$, one can obtain that

$$
Q(\varphi(t))<-\frac{\sigma_{2}}{\sigma_{1}} \quad \text { for } \quad 0 \leq t<T\left(\varphi_{0}\right) .
$$

The proof is complete by setting $\sigma_{3}:=\frac{\sigma_{2}}{\sigma_{1}}$.

THEOREM 5.11. For all minimal action solutions $v$ satisfying (5.1), if $3+\frac{4}{N} \leq$ $p<2 \cdot 2^{*}-1$ and $v$ satisfies

$$
\begin{gathered}
(4-N(p-1)) \int|\nabla v|^{2}+(4+N(p-1)) \int|x|^{2}|v|^{2} \\
+\left.\left.\left((N+2)^{2}-\frac{N(N+2)(p-1)}{2}\right) \int|\nabla| v\right|^{2}\right|^{2}<0,
\end{gathered}
$$

then the standing wave $e^{i \omega t} v(x)$ is unstable. 
Proof. Since $v$ is a minimal action solution (5.1), we know that Lemma 5.5, Lemma 5.7, Lemma 5.8 and Lemma 5.10 hold for this $v$. Note that from Lemma $5.5, Q(v)=0$ and

$$
\begin{aligned}
\left.\frac{\partial^{2}}{\partial \lambda^{2}} E\left(v^{\lambda}\right)\right|_{\lambda=1}= & \left(|\nabla v|^{2}+3|x|^{2}|v|^{2}+\left.\left.\frac{N+2}{2}(N+1)|\nabla| v\right|^{2}\right|^{2}\right) \\
& -\frac{N(p-1)}{2(p+1)}\left(\frac{N}{2}(p-1)-1\right) \int|v|^{p+1}
\end{aligned}
$$

We obtain that

$$
\begin{array}{r}
\left.\frac{\partial^{2}}{\partial \lambda^{2}} E\left(v^{\lambda}\right)\right|_{\lambda=1}=\frac{4-N(p-1)}{2} \int|\nabla v|^{2}+\frac{4+N(p-1)}{2} \int|x|^{2}|v|^{2} \\
+\left.\left.\left(\frac{(N+2)^{2}}{2}-\frac{N(N+2)(p-1)}{4}\right) \int|\nabla| v\right|^{2}\right|^{2} .
\end{array}
$$

It is now deduced from (5.17) that

$$
\left.\frac{\partial^{2}}{\partial \lambda^{2}} E\left(v^{\lambda}\right)\right|_{\lambda=1}<0 .
$$

In addition, from direct calculations, one has that $E\left(v^{\lambda}\right)<E(v)$ for $\lambda>1$ and $Q\left(v^{\lambda}\right)<Q(v)=0$ for $\lambda>1$ and $\left\|v^{\lambda}\right\|_{2}=\|v\|_{2}$. Hence $v^{\lambda} \in \Pi$ as $\lambda>1$. Since $H^{k}$ is dense in $X_{1} \cap C^{1}\left(\mathbb{R}^{N}\right)$, one has a $\varphi_{0} \in \Pi \cap H^{k}$. Assuming that the solution $\varphi(t)$ with initial data $\varphi_{0}$ exists for all time, we obtain from Lemma 5.10 that there exists $\sigma_{3}>0$ such that the solution $\varphi(t)$ of Eq.(1.1) corresponding with the initial datum $\varphi_{0}$ satisfies

$$
Q(\varphi(t))<-\sigma_{3} \quad \text { for } \quad 0 \leq t<T\left(\varphi_{0}\right)
$$

It is now deduced from Proposition 1 that $D^{\prime \prime}(t)=8 Q(\varphi(t))<-8 \sigma_{3}$. Using the standard argument of Glassey [15], we know that the standing wave $e^{i \omega t} v(x)$ is unstable.

\section{Concluding remarks and open questions}

In this concluding section, we give several remarks and questions.

Remark 6.1. In this remark, we prove that under what conditions, the $\gamma$ obtained in (4.5) is not zero. The proof is divided into two steps. In the first step, we verify for which $\Lambda$ in (4.1), $m_{\Lambda}<0$. To attain this goal, we have firstly from [27] that for $4 \leq p+1<2 \cdot 2^{*}$, the following elliptic equation

$$
-\triangle u+|x|^{2} u+u-2\left(\triangle|u|^{2}\right) u=|u|^{p-1} u
$$

has a minimal action solution $w \neq 0$ and $w \in X_{1}$. Hence $e^{i t} w(x)$ is a standing wave of Eq.(1.1). Secondly for $2<p+1<2 \cdot 2^{*}$, we know from [26] that there is a sequence of $\theta_{j} \rightarrow \infty$, such that for any $\theta_{j}$ the following equation

$$
-\triangle u+|x|^{2} u+u-2\left(\triangle|u|^{2}\right) u=\theta_{j}|u|^{p-1} u
$$

has a minimal action solution $w_{\theta_{j}} \neq 0$ and $w_{\theta_{j}} \in X_{1}$. Re-scaling these sequence of solutions by $u(x):=u\left(\theta_{j}^{-1 / 2} x\right)$ we may assume that for a sequence of positive numbers $a_{j} \rightarrow 0$ there is a sequence of solutions of

$$
-\triangle u+a_{j}^{2}|x|^{2} u+a u-2\left(\triangle|u|^{2}\right) u=|u|^{p-1} u \text {. }
$$


Let $w_{0}$ be the solution corresponding to some $0<a_{0} \leq 1$. We have the following proposition.

Proposition 2. If

$$
b=\max \left\{\frac{1}{p+1} 2^{\frac{p^{2}-2 p+5}{4}}, \frac{1}{a_{0}}\right\}
$$

and $\Lambda$ satisfies

$\left(A_{\Lambda}\right) \begin{cases}\left(\frac{p+1}{2}\right)^{\frac{2}{p-1}} b^{-\frac{N(p-1)-4}{2(p-1)}} \leq \frac{2 \Lambda}{\int\left|w_{0}\right|^{2}} \leq\left(\frac{4}{p+1} b^{\left.\frac{N(p-3)-4}{4}\right)^{\frac{2}{3-p}}}\right. & \text { if } 1<p<3, \\ \Lambda \geq \frac{1}{2} \int|w|^{2}\left(\frac{p+1}{2}\right)^{\frac{2}{p-1}} & \text { if } \quad p=3, \\ \Lambda \geq \frac{1}{2} \int|w|^{2} \max \left\{\left(\frac{p+1}{2}\right)^{\frac{2}{p-1}},\left(\frac{p+1}{4}\right)^{\frac{2}{p-3}}\right\} & \text { if } \quad 3<p<3+\frac{4}{N},\end{cases}$

then $m_{\Lambda}<0$, where $m_{\Lambda}$ is defined in (4.1).

Proof. We firstly consider the case of $3 \leq p<3+\frac{4}{N}$. Since $w$ be the minimal action solution of (6.1), we choose $s$ such that $\frac{1}{2} \int|s w|^{2}=\Lambda$, which implies that $s^{2}=\frac{2 \Lambda}{\int|w|^{2}}$. Note that

$$
\begin{aligned}
F(s w)= & \frac{s^{2}}{2} \int\left(|\nabla w|^{2}+|x|^{2}|w|^{2}\right)+\left.\left.\frac{s^{4}}{2} \int|\nabla| w\right|^{2}\right|^{2}-\frac{s^{p+1}}{p+1} \int|w|^{p+1} \\
= & \left(\frac{s^{2}}{2}-\frac{s^{p+1}}{p+1}\right) \int\left(|\nabla w|^{2}+|x|^{2}|w|^{2}\right)-\frac{s^{p+1}}{p+1} \int|w|^{2} \\
& +\left.\left.\left(\frac{s^{4}}{2}-\frac{2 s^{p+1}}{p+1}\right) \int|\nabla| w\right|^{2}\right|^{2} .
\end{aligned}
$$

One obtains that $F(s(w)<0$ if

$$
\Lambda \geq \frac{1}{2} \int|w|^{2}\left(\frac{p+1}{2}\right)^{\frac{2}{p-1}} \quad \text { for } \quad p=3
$$

and

$$
\Lambda \geq \frac{1}{2} \int|w|^{2} \max \left\{\left(\frac{p+1}{2}\right)^{\frac{2}{p-1}},\left(\frac{p+1}{4}\right)^{\frac{2}{p-3}}\right\} \quad \text { for } \quad 3<p<3+\frac{4}{N} .
$$

Secondly we deal with the case $1<p<3$. For $b>0$, define $w_{b}(x)=$ $b^{\frac{1}{p-1}} w_{0}\left(b^{\frac{1}{2}} x\right)$ and then $w_{b}$ is a solution of

$$
-\triangle u+b^{2} a_{0}^{2}|x|^{2} u+b a_{0} u-2 b^{-\frac{2}{p-1}}\left(\triangle|u|^{2}\right) u=|u|^{p-1} u
$$

Choosing $s>0$ such that $\frac{1}{2} \int\left|s w_{b}\right|^{2}=\Lambda$, then we have

$$
s=\left(\frac{2 \Lambda}{\int\left|w_{0}\right|^{2}}\right)^{\frac{1}{2}} b^{\frac{N(p-1)-4}{4(p-1)}} .
$$

Using the fact that $w_{b}$ satisfies (6.4), we have that

$$
\begin{aligned}
F\left(s w_{b}\right)= & \frac{s^{2}}{2} \int\left(\left|\nabla w_{b}\right|^{2}+|x|^{2}\left|w_{b}\right|^{2}\right)+\left.\left.\frac{s^{4}}{2} \int|\nabla| w_{b}\right|^{2}\right|^{2}-\frac{s^{p+1}}{p+1} \int\left|w_{b}\right|^{p+1} \\
= & \left(\frac{s^{2}}{2}-\frac{s^{p+1}}{p+1}\right) \int\left|\nabla w_{b}\right|^{2}+\left(\frac{s^{2}}{2}-\frac{s^{p+1}}{p+1}\right) b^{2} a_{0}^{2} \int|x|^{2}\left|w_{b}\right|^{2} \\
& +\left.\left.\left(\frac{s^{4}}{2}-\frac{2 s^{p+1}}{p+1} b^{-\frac{2}{p-1}}\right) \int|\nabla| w\right|^{2}\right|^{2}-\frac{s^{p+1}}{p+1} b a_{0} \int\left|w_{b}\right|^{2} .
\end{aligned}
$$


Hence $F\left(s w_{b}\right)<0$ if

$$
\left\{\begin{aligned}
\frac{p+1}{2} & \leq\left(\frac{2 \Lambda}{\int\left|w_{0}\right|^{2}}\right)^{\frac{p-1}{2}} b^{\frac{N(p-1)-4}{4}} \\
\Lambda & \geq \frac{1}{2} \int\left|w_{0}\right|^{2}\left(\frac{p+1}{2}\right)^{\frac{2}{p-1}} b^{-\frac{N(p-1)-4}{2(p-1)}} a_{0}^{-\frac{4}{p-1}} b^{-\frac{4}{p-1}} \\
\Lambda & \leq \frac{1}{2} \int\left|w_{0}\right|^{2}\left(\frac{4}{p+1}\right)^{\frac{2}{3-p}} b^{-\frac{2}{p-1}}
\end{aligned}\right.
$$

These inequalities hold under our assumptions on $\Lambda$ in the case of $1<p<3$. Since we have considered all cases, we prove that $m_{\Lambda}<0$.

In the second step, we show under what conditions, the $\gamma$ obtained in (4.5) is not zero.

Proposition 3. $\gamma \neq 0$ provided one of the following conditions holds:

(i): $m_{\Lambda}<0, N \geq 1$ and $3 \leq p<3+\frac{4}{N}$;

(ii): $m_{\Lambda}<0, N=2$ and $2 \leq p<3+\frac{4}{N}$.

Proof. We first prove case (i). Arguing by a contradiction, we assume that $\gamma=0$ in this case. Let $u_{0}$ be a minimizer of $m_{\Lambda}$. Then we have $\int\left(\left|\nabla u_{0}\right|^{2}+\right.$ $\left.|x|^{2}\left|u_{0}\right|^{2}+\left.\left.2|\nabla| u_{0}\right|^{2}\right|^{2}\right)=\int\left|u_{0}\right|^{p+1}$. Since $3 \leq p<3+\frac{4}{N}$, we have

$$
\begin{aligned}
F\left(u_{0}\right)= & \frac{1}{2} \int\left(\left|\nabla u_{0}\right|^{2}+|x|^{2}\left|u_{0}\right|^{2}+\left.\left.|\nabla| u_{0}\right|^{2}\right|^{2}\right) \\
& -\frac{1}{p+1} \int\left(\left|\nabla u_{0}\right|^{2}+|x|^{2}\left|u_{0}\right|^{2}+\left.\left.2|\nabla| u_{0}\right|^{2}\right|^{2}\right) \\
= & \left(\frac{1}{2}-\frac{1}{p+1}\right) \int\left(\left|\nabla u_{0}\right|^{2}+|x|^{2}\left|u_{0}\right|^{2}\right)+\left.\left.\left(\frac{1}{2}-\frac{2}{p+1}\right) \int|\nabla| u_{0}\right|^{2}\right|^{2}>0
\end{aligned}
$$

which is a contradiction to $F\left(u_{0}\right)=m_{\Lambda}<0$.

Next, we prove case (ii). Let $u_{0}$ be a minimizer of $m_{\Lambda}$. If $\gamma=0$, on one hand we have

$$
\int\left(\left|\nabla u_{0}\right|^{2}+|x|^{2}\left|u_{0}\right|^{2}+\left.\left.2|\nabla| u_{0}\right|^{2}\right|^{2}\right)=\int\left|u_{0}\right|^{p+1}
$$

On the other hand, using an argument similar to the proof of Lemma 5.5, we have that

$$
\int\left(\left|\nabla u_{0}\right|^{2}-|x|^{2}\left|u_{0}\right|^{2}+\left.\left.\frac{N+2}{2}|\nabla| u_{0}\right|^{2}\right|^{2}\right)=\frac{N(p-1)}{2(p+1)} \int\left|u_{0}\right|^{p+1}
$$

For $N=2,(6.5)$ and (6.6) imply that

$$
\left.\left.\int|\nabla| u_{0}\right|^{2}\right|^{2}=\frac{p}{2} \int|x|^{2}\left|u_{0}\right|^{2}-\frac{1}{2} \int\left|\nabla u_{0}\right|^{2}
$$


Combining this with the assumption $2 \leq p<3+\frac{4}{N}$, we deduce that

$$
\begin{aligned}
F\left(u_{0}\right)= & \frac{1}{2} \int\left(\left|\nabla u_{0}\right|^{2}+|x|^{2}\left|u_{0}\right|^{2}+\left.\left.|\nabla| u_{0}\right|^{2}\right|^{2}\right) \\
& -\frac{1}{p+1} \int\left(\left|\nabla u_{0}\right|^{2}+|x|^{2}\left|u_{0}\right|^{2}+\left.\left.2|\nabla| u_{0}\right|^{2}\right|^{2}\right) \\
= & \left(\frac{1}{2}-\frac{1}{p+1}\right) \int\left(\left|\nabla u_{0}\right|^{2}+|x|^{2}\left|u_{0}\right|^{2}\right)+\left.\left.\left(\frac{1}{2}-\frac{2}{p+1}\right) \int|\nabla| u_{0}\right|^{2}\right|^{2} \\
= & \frac{1}{4} \int\left|\nabla u_{0}\right|^{2}+\left(\frac{p}{4}-\frac{1}{2}\right) \int|x|^{2}\left|u_{0}\right|^{2}>0,
\end{aligned}
$$

which contradicts to $F\left(u_{0}\right)=m_{\Lambda}<0$. This proves $\gamma \neq 0$ in this case.

Remark 6.2. We do not know whether $\gamma \neq 0$ for any $\Lambda>0, N \geq 1$ and any $1<p<3+\frac{4}{N}$. We believe that this is a complicated problem due to the quasilinear term.

Remark 6.3. We verify that in which case the (5.17) in Theorem 5.11 holds. Let $v$ be the minimal action solution of Eq. $(5.2)$, so $Q(v)=0$ and $I(v)=0$. Denote

$$
\begin{gathered}
R(v, N)=(4-N(p-1)) \int|\nabla v|^{2}+(4+N(p-1)) \int|x|^{2}|v|^{2} \\
+\left.\left.\left((N+2)^{2}-\frac{N(N+2)(p-1)}{2}\right) \int|\nabla| v\right|^{2}\right|^{2} .
\end{gathered}
$$

Case I. If $N=2$, we have that

$$
\begin{gathered}
\int|\nabla v|^{2}-\int|x|^{2}|v|^{2}+\left.\left.2 \int|\nabla| v\right|^{2}\right|^{2}=\frac{p-1}{p+1} \int|v|^{p+1} \\
\int|\nabla v|^{2}+\omega \int|v|^{2}+\int|x|^{2}|v|^{2}+\left.\left.2 \int|\nabla| v\right|^{2}\right|^{2}=\int|v|^{p+1} .
\end{gathered}
$$

It is deduced from (6.7) and (6.8) that

$$
\left.\left.\int|\nabla| v\right|^{2}\right|^{2}=-\frac{1}{2} \int|\nabla v|^{2}+\frac{p}{2} \int|x|^{2}|v|^{2}+\frac{p-1}{2} \omega \int|v|^{2}
$$

Replacing (6.9) into $R(v, 2)$, we get that

$$
R(v, 2)=-4 \int|\nabla v|^{2}-2\left(p^{2}-6 p-1\right) \int|x|^{2}|v|^{2}+(10-2 p)(p-1) \omega \int|v|^{2} .
$$

Therefore if $\omega>0$ and $p \geq 3+\sqrt{10}$, then $R(v, 2)<0$. Thus condition (5.17) can be verified in the case of $\omega>0, N=2$ and $p \geq 3+\sqrt{10}$.

Case II. If $N=4$, then we have from $Q(v)=0$ and $I(v)=0$ that

$$
\begin{aligned}
& \int|\nabla v|^{2}-\int|x|^{2}|v|^{2}+\left.\left.3 \int|\nabla| v\right|^{2}\right|^{2}=\frac{2(p-1)}{p+1} \int|v|^{p+1} \\
& \int|\nabla v|^{2}+\omega \int|v|^{2}+\int|x|^{2}|v|^{2}+\left.\left.2 \int|\nabla| v\right|^{2}\right|^{2}=\int|v|^{p+1} .
\end{aligned}
$$

Thus, we obtain from (6.10) and (6.11) that

$$
\begin{aligned}
\left.\left.\int|\nabla| v\right|^{2}\right|^{2}= & \left(\frac{p+1}{2(7-p)}-\frac{1}{2}\right) \int|\nabla v|^{2}+\left(\frac{3(p+1)}{2(7-p)}-\frac{1}{2}\right) \omega \int|v|^{2} \\
& +\left(\frac{5(p+1)}{2(7-p)}-\frac{1}{2}\right) \int|x|^{2}|v|^{2} .
\end{aligned}
$$


Substitute (6.12) into $R(v, 4)$ we get that

$$
\begin{aligned}
& R(v, 4)=\left((8-4 p)+\frac{12(4-p)(p-3)}{2(7-p)}\right) \int|\nabla v|^{2} \\
& \quad+\frac{4}{7-p}\left(-10 p^{2}+46 p-12\right) \int|x|^{2}|v|^{2}+\frac{24(4-p)(p-1)}{7-p} \omega \int|v|^{2} .
\end{aligned}
$$

Therefore, if $\omega>0$ and $\frac{23}{10}+\sqrt{\frac{409}{100}} \leq p<7$, then $R(v, 4)<0$. Hence, condition (5.17) can be verified in the case of $\omega>0, N=4$ and $\frac{23}{10}+\sqrt{\frac{409}{100}} \leq p<7$ (since when $\left.N=4,2 \cdot 2^{*}-1=7\right)$.

Remark 6.4. From the previous section, we know that $3+\frac{4}{N}$ seems to be a critical exponent of (1.1). For the semilinear equation (1.2), the critical exponent is $1+\frac{4}{N}$. The harmonic potential term $|x|^{2} \varphi$ makes the existence of stable standing waves $e^{i \mu_{n} t} u(x)$ of (1.2) in the case of $1+\frac{4}{N}<p<2^{*}-1$, where $\mu_{n} \rightarrow \mu_{0}$, see $[32,13]$ for details. It also makes the standing waves $e^{i \omega t} u(x)$ be unstable for any frequency $\omega>0$ and suitable bigger $p$. The previous remark shows that for (1.1), the harmonic potential term $|x|^{2} \varphi$ seems to play similar roles. However, due to the quasilinear term, detailed characterizations of these are open.

Remark 6.5. We point out that if one replaces the perturbation $\left(\triangle|\varphi|^{2}\right) \varphi$ in (1.1) by a more general term $\left(\triangle|\varphi|^{2 \alpha}\right)|\varphi|^{2 \alpha-2} \varphi$ with $\alpha>\frac{1}{2}$, one can also get the stable and unstable standing wave by these methods. Indeed, one can prove that the existence of stable standing wave for $1<p<4 \alpha-1+\frac{4}{N}$ and the unstable standing wave for $4 \alpha-1+\frac{4}{N} \leq p<2 \alpha \cdot 2^{*}-1$. We left the details to the interested readers. Acknowledgments. This work was partially done during the first author staying in the Department of Mathematics, University of Aveiro, Portugal. He thanks the warm hospitality of all the members of the department. J. Chen thanks Professor Z.-Q. Wang for some helpful discussions and comments. This work was financially supported by NSF of China (10971026), NSF of Fujian (2009J06001) and the Portuguese Foundation for Science and Technology (FCT) and the research unit CIDMA.

\section{References}

[1] T. Bartsch and Z.-Q. Wang, Existence and multiplicity results for some superlinear elliptic problems on $\mathbb{R}^{N}$, Comm. Partial Differential Equations 20 (1995), 1725-1741.

[2] H. Berestycki and T. Cazenave, Instabilite des etats stationnaires dans les equations de schrodinger et de Klein-Gordon non linearires, C. R. Acad. Sci. Paris, Serie I 293 (1981), 489-492.

[3] A. de Bouard, N. Hayashi and J. C. Saut, Global existence of small solutions to a relativistic nonlinear Schrödinger equation, Commun. Math. Phys. 189 (1997), 73-105.

[4] H. Brezis and E. Lieb, A relation between pointwise convergence of functions and convergence of functionals, Proc. Amer. Math. Soc. 88 (1983), 486-490.

[5] F. E. Browder, Variational methods for nonlinear elliptic eigenvalue problems, Bull. Amer. Math. Soc. 71 (1965), 176-183.

[6] T. Cazenave, Semilinear Schrödinger Equations, Courant Institute of Mathematical Sciences, Vol. 10, Providence, Rhode Island, 2005.

[7] T. Cazenave and P. L. Lions, Orbital stability of standing waves for some nonlinear Schrödinger equations, Commun. Math. Phys. 85 (1982), 549-561.

[8] X. L. Chen and R. N. Sudan, Necessary and sufficient conditions for self-focusing of shortultrains tense laser pulse in underdense plasma, Phys. Rev. Let. 70 (1993), 2082-2085.

[9] J. Chen and B. Guo, Blow-up and strong instability result for a quasilinear Schrödinger equation, Appl. Math. Model. 33 (2009), 4192-4200. 
[10] J. Chen, Y. Li and Z.-Q. Wang, Stability of standing waves for a class of quasilinear Schrödinger equations, Submitted.

[11] M. Colin and L. Jeanjean, Solutions for a quasilinear Schröinger equation: a dual approach, Nonlinear Anal. TMA 56 (2004), 213-226.

[12] M. Colin, L. Jeanjean, and M. Squassina, Stability and instability results for standing waves of quasi-linear Schrödinger equations, Nonlinearity 23 (2010), 1353-1385.

[13] R. Fukuizumi, Stability and instability of standing waves for the nonlinear Schrödinger equation with harmonic potential, Discrete Contin. Dyn. Syst. 7 (2001), 525-544.

[14] R. Fukuizumi and M. Ohta, Stability of standing waves for nonlinear Schrödinger equations with potentials, Differential Integral Equations 16 (2003), 111-128.

[15] R. T. Glassey, On the blowing-up of solutions to the Cauchy problem for the nonlinear Schrödinger equation, J. Math. Phys. 18 (1977), 1794-1797.

[16] M. Grillakis, J. Shatah and W. Strauss, Stability theory of solitary waves in the presence of symmetry I, J. Functional Analysis 74 (1987), 160-197.

[17] M. Grillakis, J. Shatah and W. Strauss, Stability theory of solitary waves in the presence of symmetry II, J. Functional Analysis 94 (1990), 308-348.

[18] B. Guo and J. Chen, Orbital stability of standing wave solution for a quasilinear Schrodinger equation, Quart. Appl. Math. 67 (2009), 781-791.

[19] B. Guo, J. Chen and F. Su, The "Blow up" problem for a quasilinear Schrödinger equation, J. Math. Phys. 46 (2005), 073510, 1-10.

[20] R. W. Hasse, A general method for the solution of nonlinear soliton and kink Schrödinger equations, Z. Physik B 37 (1980), 83-87.

[21] I. D. Iliev and K. P. Kirchev, Stability and instability of solitary waves for one-dimensional singular Schrödinger equations, Differential Integral Equations 6 (1993), 685-703.

[22] C. E. Kenig, G. Ponce and L. Vega, The Cauchy problem for quasilinear Schrödinger equations, Invent. Math. 158 (2004), 343-388.

[23] S. Kurihura, Large amplitude quasi-solitons in superfluid film, J. Phys. Soc. Japan 50 (1981), 3262-3267.

[24] H. Lange, M. Poppenperg and H. Teismann, Nash-Moser methods for the solutions of quasilinear Schrödinger equations, Comm. Partial Differential Equations 24 (1999), 1399-1418.

[25] E. H. Lieb and M. Loss, Analysis, Graduate Studies in Mathematics 14, Providence, Rhode Island, 2001.

[26] J. Q. Liu and Z.-Q. Wang, Soliton solutions for quasilinear Schrödinger equations, Proc. Amer. Math. Soc. 131 (2003), 486-490.

[27] J. Q. Liu, Y. Wang and Z.-Q. Wang, Solutions for quasilinear Schrödinger equations via Nehari method, Comm. Partial Differential Equations 29 (2004) 879-901.

[28] M. Poppenberg, On the local well posedness of quasilinear Schrödinger equations in arbitrary space dimension, J. Differential Equations 172 (2001), 83-115.

[29] M. Poppenberg, K. Schmitt and Z.-Q. Wang, On the existence of soliton solutions to quasilinear Schrödinger equations, Calc. Var. Partial Differential Equations 14 (2002), 329-344.

[30] M. Porkolab and M. V. Goddman, Upper hybrid solitons and oscillating two-stream instabilities, Phys. Fluids 19 (1976), 872-881.

[31] P. Rabinowitz, On a class of nonlinear Schrödinger equations, Z. Angew. Math. Phys. 43 (1992), 270-291.

[32] H. A. Rose and M. I. Weinstein, On the bound states of the nonlinear Schrödinger equation with linear potential, Phys. D. 30 (1988), 207-218.

[33] J. Shatah and W. Strauss, Instability of nonlinear bound states, Commun. Math. Phys. 100 (1985), 173-190.

[34] K.H. Spatschek and S.G.Tagary, Nonlinear propagation of ion-cyclotron modes, Phys. Fluids 20 (1977), 1505-1509.

[35] W. Strauss, Existence of solitary waves in higher dimensions, Commun. Math. Phys. 55 (1977), 149-162.

[36] C. A. Stuart, Lectures on the orbital stability of standing waves and application to the nonlinear Schrödinger, Milan J. Math. 76 (2008), 329-399.

[37] M. Y. Yu and P. K. Shuhla, On the formation of upper-hybrid solitons, Plasma Phys. 19 (1977), 889-893.

[38] M. I. Weinstein, Nonlinear Schrödinger equations and sharp interpolation estimates, Commun. Math. Phys. 87 (1983), 567-576. 
[39] J. Zhang, Stability of attractive Bose-Einstein condensates, J. Stat. Phys. 102 (2000), 731746.

[40] J. Zhang, Stability of standing waves for nonlinear Schrödinger equation with unbounded potentials, Z. Angew. Math. Phys. 51 (2000), 498-503.

School of Mathematics and Computer Science, Fujian Normal University, Fuzhou 350007, P. R. China; and Department of Mathematics, University of Aveiro, 3810-193 Aveiro, Portugal

Department of Mathematics, University of Aveiro, 3810-193 Aveiro, Portugal 